\title{
NONLINEAR SEMIGROUPS AND THE EXISTENCE AND STABILITY OF SOLUTIONS OF SEMILINEAR NONAUTONOMOUS EVOLUTION EQUATIONS
}

\author{
BERND AULBACH AND NGUYEN VAN MINH
}

\begin{abstract}
This paper is concerned with the existence and stability of solutions of a class of semilinear nonautonomous evolution equations. A procedure is discussed which associates to each nonautonomous equation the so-called evolution semigroup of (possibly nonlinear) operators. Sufficient conditions for the existence and stability of solutions and the existence of periodic oscillations are given in terms of the accretiveness of the corresponding infinitesimal generator. Furthermore, through the existence of integral manifolds for abstract evolutionary processes we obtain a reduction principle for stability questions of mild solutions. The results are applied to a class of partial functional differential equations.
\end{abstract}

\section{INTRODUCTION}

In the last three decades the theory of semigroups of nonlinear operators has been developed extensively and the achieved results have found many applications in the theory of partial differential equations (see the survey [11] by M.G. Crandall). Recently, increasing interest has been observed in applications of the methods of dynamical systems to infinite dimensional dynamics (see, e.g., [9], [7], [8], [15], [18], [26], [28] and the references therein). The main idea in this context is to associate a semigroup of nonlinear operators to an evolution equation and then to study the asymptotic behavior of the solutions of this equations in the vicinity of a given stationary solution. Whereas most of those papers deal only with autonomous, i.e. time independent evolution equations, the explicit time dependence of evolution

1991 Mathematics Subject Classification. 34G20, 34K30, 47H20.

Key words and phrases. Evolutionary process, evolution semigroup, semilinear nonautonomous equation, nonlinear semigroup, stability, periodic solution, accretive operator, integral manifold, instability.

The second author was on leave (as a research fellow of the Alexander von Humboldt Foundation) from the Department of Mathematics, University of Hanoi, Vietnam.

Received: July 25, 1996. 
equations often arises quite naturally, not only in physics and mechanics, but also in mathematics when one linearizes an autonomous equation along a nonstationary solution. For particular classes of time-dependent evolution equations arising from the linearization along a compact invariant subset it has been shown (see e.g. [43]) that one can define a skew-product semiflow which allows to apply the methods of classical dynamical systems to the underlying nonautonomous equations.

To the best of our knowledge, the papers [32, 33] contain the first attempt to associate a strongly continuous evolution semigroup to a nonlinear timedependent equation in order to study the asymptotic behavior of solutions. Since the present paper is closely related to those articles we briefly recall some basic results proved in $[32,33]$. The right hand sides of the equations considered are defined everywhere and they are supposed to be Lipschitz continuous. To each equation of this kind one associates an evolution semigroup with properties which allow to apply the Crandall-Liggett theorem on the generation of nonlinear semigroups. In a recent paper [2] we considered equations with almost periodic coefficients in this semigroup framework. The main obstacles for the application of those results to infinite dimensional systems are apparently due to the assumption that the right hand sides of the equations considered are Lipschitz continuous and that they are defined everywhere.

In this paper we are concerned with evolution equations of the form

$$
\frac{d x}{d t}=A(t) x+f(t, x)
$$

where $A(t)$ is a (possibly unbounded) linear operator acting in a real or complex Banach space $\mathbf{X}$ and $f(\cdot, \cdot): \mathbb{R} \times \mathbf{X} \rightarrow \mathbf{X}$ is a (possibly nonlinear) continuous function. We furthermore assume that the linear part $d x / d t=$ $A(t) x$ of equation (1) is well posed in a sense to be explained. To this kind of equation we manage to associate an evolution semigroup which is strongly continuous and whose generator can be computed explicitly in terms of the generator of the evolution semigroup associated with the linear part of (1) and the nonlinear term $f(t, x)$. Finally, we discuss how to apply this semigroup approach to the study of the asymptotic behavior of mild solutions of equation (1).

For the case of a time independent linear part of equation (1) the existence problem for solutions has been investigated by many authors (see e.g. [21], [24], [27], [35], [36], [37], [38], [48] and the references therein). In the present paper we show that the problems arising from the explicit $t$-dependence of $A(t)$ can be overcome by using our evolution semigroup approach. Furthermore, in the study of the asymptotic behavior of mild solutions of equation (1) this approach allows to make use of many results available for dynamical systems.

A more detailed outline of our construction is as follows. First we associate to equation (1) with Lipschitz continuous $f(t, x)$ a strongly continuous evolution semigroup whose generator is of the form $A+F$, where $A$ is the 
generator of the linear evolution semigroup associated with the linear part of equation (1) and $F$ is an operator acting on a function space induced by $f(\cdot, \cdot)$. Without any additional assumption on the linear part we do not know any relation between the associated evolution semigroup and the semigroup (if there exists any at all) generated by $A+F$ in the Crandall-Liggett sense. Nevertheless, using an appropriate adaptation of a fundamental result due to G.F. Webb [48] for time independent equations we manage to prove that mild solutions of equation (1) exist and that the semigroup generated by $A+F$ in Webb's sense coincides with the evolution semigroup associated with equation (1). In order to accomplish this we first solve the corresponding equation with right hand side $A+F$ in a suitable function space by using Webb's generation theorem (see [48]), and then we consider equation (1). In doing so we can prove the existence and uniqueness of mild solutions and the coincidence of the semigroup generated by $A+F$ in Webb'sense with the evolution semigroup associated with equation (1). This result is a substantial generalization of a major result on nonlinear equations obtained in [33]. It turns out that for equation (1) with $\tau$-periodic coefficients the evolution operator $S^{\tau}$ from the evolution semigroup acts like a Poincaré mapping. This analogy provides a sufficient condition for the existence of $\tau$-periodic mild solutions of equation (1) in terms of the accretiveness of $A$ and $F$. In order to study the instability of mild solutions of (1) we prove in Section 3 a theorem on the existence of integral manifolds for evolutionary processes by using the Hadamard graph transform. Since this result is derived in a very general setting (without use of any concrete equations) it is applicable to various kinds of equations. An application to partial functional differential equations is presented in Section 4.

\section{Evolution SEmigroups: EXISTEnCE AND STABility of SOlutions}

In this section we consider the evolution semigroups associated with evolutionary processes defined by semilinear equations. One of the main topics to be discussed here is the description of the infinitesimal generators and their use in getting sufficient conditions for the existence and stability of solutions of equation (1).

We first introduce some definitions and notations which will be used throughout this paper. Without further mention, $\mathbf{X}$ will always be a given real or complex Banach space. By $L_{p}(\mathbf{X}), 1 \leq p<\infty$ we denote the space of all (equivalence classes of) $\mathbf{X}$-valued measurable functions $v$ on $\mathbb{R}$ such that $\int_{\mathbb{R}}\|v(t)\|^{p} d t<\infty$ with norm $\|\cdot\|_{p}$. The integral is always to be understood in the Bochner sense (see e.g. [50]). By $C_{u}(\mathbb{R}, \mathbf{X})$ we mean the space of all bounded, uniformly continuous functions from $\mathbb{R}$ to $\mathbf{X}$ equipped with the supremum norm, while $C_{0}(\mathbf{X})$ denotes the subspace of functions $w \in C_{u}(\mathbb{R}, \mathbf{X})$ with the property $\lim _{|t| \rightarrow \infty}\|w(t)\|=0$. Various notions of stability and instability will be used in a standard sense (see e.g. [13]). 
Definition 1. A family $\{X(t, s) \mid t, s \in \mathbb{R}, t \geq s\}$ of (possibly nonlinear) operators acting on $\mathbf{X}$ is called an evolutionary process if it satisfies the following conditions:

(i) $X(s, s) x=x \quad$ for all $s \in \mathbb{R}, x \in \mathbf{X}$;

(ii) $X(t, s) X(s, r)=X(t, r)$ for all $t \geq s \geq r$.

Such an evolutionary process is called continuous if it satisfies the conditions

(iii) $\|X(t, s) x-X(t, s) y\| \leq K e^{\omega(t-s)}\|x-y\| \quad$ for all $t \geq s$ and $x, y \in \mathbf{X}$, where $K$ is any positive and $\omega$ any real constant,

(iv) $X(t, s) x$ is continuous jointly with respect to $t, s$ and $x$.

To every evolutionary process $\{X(t, s) \mid t \geq s\}$ we associate the so-called evolution semigroup $\left\{T^{h} \mid h \geq 0\right\}$ defined by the relation

$$
\left(T^{h} v\right)(t)=X(t, t-h) v(t-h) \quad \text { for all } t \in \mathbb{R}
$$

where $v$ belongs to a suitable space of functions (such as the ones mentioned above).

Proposition 1. Assume that $\{X(t, s) \mid t \geq s\}$ is a continuous evolutionary process such that $X(t, s) 0=0$ for all $t \geq s$. Then for any of the function spaces $L_{p}(\mathbf{X}), 1 \leq p<\infty$, and $C_{0}(\mathbf{X})$ the associated evolution semigroup $\left\{T^{h} \mid h \geq 0\right\}$ is strongly continuous.

Proof. We give a proof for the case $L_{p}(\mathbf{X})$ only because the proof for $C_{0}(\mathbf{X})$ is essentially the same. We first notice that for every $v \in L_{p}(\mathbf{X})$ the function taking $t$ into $X(t, t-h) v(t-h)$ is measurable. Furthermore, we get

$$
\begin{aligned}
\left(\int_{\mathbb{R}}\left\|\left(T^{h} v\right)(t)\right\|^{p} d t\right)^{1 / \mathrm{p}} & =\left(\int_{\mathbb{R}}\|X(t, t-h) v(t-h)\|^{p} d t\right)^{1 / \mathrm{p}} \\
& \leq\left(\int_{\mathbb{R}}\left\|K e^{\omega h} v(t-h)\right\|^{p} d t\right)^{1 / \mathrm{p}} \leq K e^{\omega h}\|v\|_{p}<\infty .
\end{aligned}
$$

In order to prove that the semigroup $\left\{T^{h} \mid h \geq 0\right\}$ is strongly continuous we first show that the relation

$$
\lim _{h \rightarrow 0^{+}}\left(T^{h} v-v\right)=0
$$

is true for every continuous $v$ with compact support. Indeed, by assumption there exists a positive constant $N$ such that $v(t)=0$ for all $|t| \geq N-1$. Thus (3) is equivalent to

$$
\lim _{h \rightarrow 0^{+}} \int_{-N}^{N}\|X(t, t-h) v(t-h)-v(t)\|^{p} d t=0 .
$$

Since $\{X(t, s) \mid t \geq s\}$ and $v$ are continuous, the function $\|X(t, t-h) v(t-h)\|$ attains its maximum as $(t, h)$ varies in $[-N, N] \times[0,1]$. Thus, the claimed 
relation (3) is a consequence of the following estimate which uses the FatouLebesgue Lemma:

$$
\begin{aligned}
0 & =\int_{-N}^{N} \limsup _{h \rightarrow 0^{+}}\|X(t, t-h) v(t-h)-v(t)\|^{p} d t \geq \\
& \geq \limsup _{h \rightarrow 0^{+}} \int_{-N}^{N}\|X(t, t-h) v(t-h)-v(t)\|^{p} d t \geq 0 .
\end{aligned}
$$

In order to conclude the proof of the proposition we now consider an arbitrary $v \in L_{p}(\mathbf{X})$ and choose for every positive $\epsilon$ a continuous function $w$ with compact support and the property $\|w-v\|_{p}<\epsilon$. Then we get

$$
\begin{aligned}
& \limsup _{h \rightarrow 0^{+}}\left(\int_{\mathbb{R}}\|X(t, t-h) v(t-h)-v(t)\|^{p} d t\right)^{1 / \mathrm{p}} \\
& \leq \limsup _{h \rightarrow 0^{+}}\left[\left(\int_{\mathbb{R}}\|X(t, t-h) v(t-h)-X(t, t-h) w(t-h)\|^{p} d t\right)^{1 / \mathrm{p}}\right. \\
& \quad+\left(\int_{\mathbb{R}}\|X(t, t-h) w(t-h)-w(t)\|^{p} d t\right)^{1 / \mathrm{p}} \\
& \left.\quad+\left(\int_{\mathbb{R}}\|w(t)-v(t)\|^{p} d t\right)^{1 / \mathrm{p}}\right] \\
& \leq K \epsilon+\limsup _{h \rightarrow 0^{+}}\left(\int_{\mathbb{R}}\|X(t, t-h) w(t-h)-w(t)\|^{p} d t\right)^{1 / \mathrm{p}}+\epsilon=(1+K) \epsilon .
\end{aligned}
$$

Since $\epsilon$ is arbitrary, this estimate proves (3) for every $v \in L_{p}(\mathbf{X})$ and therefore completes the proof of Proposition 1.

Remark. For the linear case the above proposition has been proved in [40].

It is known (see e.g. [12]) that nonlinear semigroups need not have infinitesimal generators even if they are strongly continuous. So in order to get generators of the evolution semigroups associated with continuous evolutionary processes we will consider processes generated by equation (1) under some additional conditions.

Definition 2. The linear equation

$$
\frac{d x}{d t}=A(t) x
$$

is said to be well-posed if there exists a continuous linear evolutionary process $\{U(t, s) \mid t \geq s\}$ such that for every $s \in \mathbb{R}$ and $x \in D(A(s))$ the function $x(t)=U(t, s) x$ is the uniquely determined solution of equation (4) satisfying $x(s)=x$.

Definition 3. Suppose the linear equation (4) is well-posed. Then every solution $x(t)$ (defined on some interval $[s, s+\delta), \delta>0$ ) of the integral 
equation

$$
x(t)=U(t, s) x+\int_{s}^{t} U(t, \xi) f(\xi, x(\xi)) d \xi, \quad t \geq s
$$

is called a mild solution of the semilinear equation (1) starting from $x$ at $t=s$. Furthermore, equation (1) is said to generate an evolutionary process $\{X(t, s) \mid t \geq s\}$ if for every $x \in \mathbf{X}$ the function $X(t, s) x, t \geq s$ is the unique solution of equation (5).

Proposition 2. Suppose the following conditions are satisfied:

(i) The linear equation (4) is well-posed.

(ii) The nonlinear function $f(t, x)$ is continuous jointly with respect to $t$ and $x$ and Lipschitz continuous with respect to $x$ uniformly in $t \in \mathbb{R}$ and $f(t, 0)=0$ for all $t \in \mathbb{R}$.

Then the semilinear equation (1) generates a continuous evolutionary process whose associated evolution semigroup on $L_{p}(\mathbf{X})$ or $C_{0}(\mathbf{X})$ is strongly continuous and has an infinitesimal generator of the form $A+F$, where $A$ is the infinitesimal generator of the linear evolution semigroup associated with the evolutionary process generated by the linear equation (4) in $L_{p}(\mathbf{X})$ or $C_{0}(\mathbf{X})$, respectively, and $F$ is the operator taking $v$ from $L_{p}(\mathbf{X})$ or $C_{0}(\mathbf{X})$, respectively, into the function $t \mapsto f(t, v(t))$.

Proof. Using standard arguments (see e.g. [45]) one can prove that equation (1) generates an evolutionary process. Furthermore, this evolutionary process is continuous. In fact, from [45] it follows that $X(t, s) x$ is continuous jointly with respect to $t, s, x$. Now we prove that $X(t, s) x$ also satisfies the Lipschitz condition (iii) in Definition 1. By definition of the evolutionary process $\{X(t, s) \mid t \geq s\}$ we get

$$
\begin{aligned}
\| X(t, s) x- & X(t, s) y \| \\
\leq & \|U(t, s) x-U(t, s) y\| \\
& +\int_{s}^{t}\|U(t, \xi)\| \cdot\|f(\xi, X(\xi, s) x)-f(\xi, X(\xi, s) y)\| d \xi \\
\leq & K e^{\omega(t-s)}\|x-y\|+\int_{s}^{t} K e^{\omega(t-\xi)} L\|X(\xi, s) x-X(\xi, s) y\| d \xi,
\end{aligned}
$$

where $L$ is a Lipschitz constant of $f(t, x)$ with respect to $x$, and $K, \omega$ stem from the well-posedness of the linear equation (4). Just for convenience we obviously may choose $\omega$ to be positive. Applying Gronwall's Lemma we get

$$
\|X(t, s) x-X(t, s) y\| \leq K e^{(\omega+K L)(t-s)}\|x-y\|, \quad t \geq s, x, y \in \mathbf{X} .
$$

Therefore the evolutionary process $\{X(t, s) \mid t \geq s\}$ is continuous according to Definition 1. Taking into account that $X(t, s) 0=0$ for all $t \geq s$ we can apply Proposition 1 to see that the associated evolution semigroup $\left\{T^{h} \mid h \geq\right.$ $0\}$ is strongly continuous. Now we are going to compute the infinitesimal generator of this semigroup. To this purpose we first prove that for every 
$w \in L_{p}(\mathbf{X})$ which is continuous and has compact support we get the relation

$$
\lim _{h \rightarrow 0^{+}} \int_{\mathbb{R}}\left\|f(t, w(t))-h^{-1} \int_{t-h}^{t} U(t, \xi) f(\xi, X(\xi, t-h) w(t-h)) d \xi\right\|^{p} d t=0 .
$$

Indeed, by definition $f(\cdot, w(\cdot))$ is uniformly continuous. Consequently we get

$$
\lim _{h \rightarrow 0^{+}} \sup _{t} \int_{t-h}^{t}\|f(\xi, w(\xi))-f(t, w(t))\| d \xi=0 .
$$

Furthermore we have

$$
\begin{aligned}
& \left(\int_{\mathbb{R}}\left\|f(t, w(t))-h^{-1} \int_{t-h}^{t} U(t, \xi) f(\xi, X(\xi, t-h) w(t-h)) d \xi\right\|^{p} d t\right)^{1 / \mathrm{p}} \\
& \leq\left(\int_{\mathbb{R}}\left\|f(t, w(t))-h^{-1} \int_{t-h}^{t} U(t, \xi) f(\xi, w(\xi)) d \xi\right\|^{p} d t\right)^{1 / \mathrm{p}} \\
& +\left(\int_{\mathbb{R}}\left\|h^{-1} \int_{t-h}^{t} U(t, \xi)[f(\xi, w(\xi))-f(\xi, X(\xi, t-h) w(t-h))] d \xi\right\|^{p} d t\right)^{1 / \mathrm{p}} .
\end{aligned}
$$

Since $w$ has compact support, from the assumptions we observe that $f(\cdot, w(\cdot))$ has compact support as well. Consequently we get

$$
\lim _{h \rightarrow 0^{+}}\left(\int_{\mathbb{R}}\left\|f(t, w(t))-h^{-1} \int_{t-h}^{t} U(t, \xi) f(\xi, w(\xi)) d \xi\right\|^{p} d t\right)^{1 / \mathrm{p}}=0 .
$$

On the other hand, we have

$$
\begin{aligned}
& \left(\int_{\mathbb{R}}\left\|h^{-1} \int_{t-h}^{t} U(t, \xi) f(\xi, w(\xi))-f(\xi, X(\xi, t-h) w(t-h)) d \xi\right\|^{p} d t\right)^{1 / \mathrm{p}} \\
& \leq\left(\int_{\mathbb{R}}\left\|h^{-1} \int_{t-h}^{t} K e^{\omega h} L\right\| w(\xi)-X(\xi, t-h) w(t-h)\|d \xi\|^{p} d t\right)^{1 / \mathrm{p}} .
\end{aligned}
$$

Note that the function $g(t, \xi, h):=\|w(\xi)-X(\xi, t-h) w(t-h)\|$ is continuous with respect to $(t, \xi, h) \in\{(t, \xi, h) \mid h \in[0,1],-N \leq t-h \leq \xi \leq N\}$, where $\operatorname{supp}(w) \subset[-N, N]$. Consequently, the function

$$
q(t, h):=\left\|h^{-1} \int_{t-h}^{t} K e^{\omega h} L\right\| w(\xi)-X(\xi, t-h) w(t-h)\|d \xi\|^{p}
$$

is bounded in $(t, h) \in[-N-1, N+1] \times[0,1]$. Now applying the FatouLebesgue Dominant Convergence Lemma we get

$$
\limsup _{h \rightarrow 0^{+}}\left(\int_{\mathbb{R}}\left\|h^{-1} \int_{t-h}^{t} K e^{\omega h} L\right\| w(\xi)-X(\xi, t-h) w(t-h)\|d \xi\|^{p} d t\right)^{1 / p}=0 .
$$

All of this implies that the claimed relation (7) has been verified under the assumption that $w$ is continuous and has compact support. 
In order to show that (7) is true for all functions from $L_{p}(\mathbf{X})$, we now choose an arbitrary element $v$ from this space as well as an arbitrary continuous function $w$ with compact support. Then, using (6) and (7) we get

$$
\begin{aligned}
& \limsup _{h \rightarrow 0^{+}}\left(\int_{\mathbb{R}} \| f(t, v(t))\right. \\
& \left.\quad-h^{-1} \int_{t-h}^{t} U(t, \xi) f(\xi, X(\xi, t-h) v(t-h)) d \xi \|^{p} d t\right)^{1 / \mathrm{p}} \\
& \leq \limsup _{h \rightarrow 0^{+}}\left(\int_{\mathbb{R}}\|f(t, v(t))-f(t, w(t))\|^{p} d t\right)^{1 / \mathrm{p}} \\
& \quad+\limsup _{h \rightarrow 0^{+}}\left(\int_{\mathbb{R}} \| f(t, w(t))\right. \\
& \left.\quad-h^{-1} \int_{t-h}^{t} U(t, \xi) f(\xi, X(\xi, t-h) w(t-h)) d \xi \|^{p} d t\right)^{1 / \mathrm{p}} \\
& \quad+\limsup _{h \rightarrow 0^{+}}\left(\int_{\mathbb{R}} \| h^{-1} \int_{t-h}^{t} U(t, \xi)[f(\xi, X(\xi, t-h) w(t-h))\right. \\
& \left.\quad-\quad f(t, X(\xi, t-h) v(t-h))] d \xi \|^{p} d t\right)^{1 / \mathrm{p}} \\
& \leq \limsup _{h \rightarrow 0^{+}} L\left(\int_{\mathbb{R}}\|v(t)-w(t)\|^{p} d t\right)^{1 / \mathrm{p}} \\
& \quad+\limsup _{h \rightarrow 0^{+}}\left(\int_{\mathbb{R}}\left\|h^{-1} \int_{t-h}^{t} K e^{\omega h} L\right\| X(\xi, t-h) w(t-h)\right. \\
& \left.\quad-X(\xi, t-h) v(t-h)\|d \xi\|^{p} d t\right)^{1 / \mathrm{p}} .
\end{aligned}
$$

Using the estimate (6) we continue this estimate to get

$$
\begin{aligned}
\leq & L\|v-w\|_{p} \\
& +\limsup _{h \rightarrow 0^{+}}\left(\int_{\mathbb{R}} \| h^{-1} K e^{\omega h}\right. \\
& \left.\quad \int_{t-h}^{t} L K e^{(\omega+K L) h}\|w(t-h)-v(t-h)\| d \xi \|^{p} d t\right)^{1 / \mathrm{p}} \\
= & L\|v-w\|_{p} \\
& +\limsup _{h \rightarrow 0^{+}}\left(L K^{2} e^{(2 \omega+K L) h} \int_{\mathbb{R}}\|w(t-h)-v(t-h)\|^{p} d t\right)^{1 / \mathrm{p}} \\
\leq & L\|v-w\|_{p}+L K^{2}\|v-w\|_{p} \\
= & \left(L+L K^{2}\right)\|w-v\|_{p} .
\end{aligned}
$$


Since $w$ in (12) is an arbitrary continuous function with compact support, (12) implies for any $v \in L_{p}(\mathbf{X})$ the claimed relation

$$
\begin{aligned}
& \lim _{h \rightarrow 0^{+}}\left(\int_{\mathbb{R}}\left\|f(t, v(t))-h^{-1} \int_{t-h}^{t} U(t, \xi) f(\xi, X(\xi, t-h) v(t-h)) d \xi\right\|^{p} d t\right)^{1 / \mathrm{p}} \\
& =0 .
\end{aligned}
$$

By the definitions of $T^{h}$ and $X(t, s)$ we obtain

$$
\begin{aligned}
\left(\frac{T^{h} v-v}{h}\right)(t)=\frac{X(t, t-h) v(t-h)-v(t)}{h} \\
=\frac{U(t, t-h) v(t-h)-v(t)}{h} \\
\quad+h^{-1} \int_{t-h}^{t} U(t, \xi) f(\xi, X(\xi, t-h) v(t-h)) d \xi \\
=\frac{U(t, t-h) v(t-h)-v(t)}{h}+f(t, v(t)) \\
\quad-\left[f\left(t, v(t)-h^{-1} \int_{t-h}^{t} U(t, \xi) f(\xi, X(\xi, t-h) v(t-h)) d \xi\right] .\right.
\end{aligned}
$$

It is clear from (13) and (14) that $v$ belongs to the domain of the infinitesimal generator of $\left\{T^{h} \mid h \geq 0\right\}$ if and only if $v$ belongs to the domain of the generator $A$ of the linear evolution semigroup associated with $\{U(t, s) \mid t \geq$ $s\}$.

Since the proof for the $C_{0}(\mathbf{X})$ case requires no essential changes, the proof of Proposition 2 is complete.

Corollary 1. Suppose the assumptions of Proposition 2 are satisfied. Then the infinitesimal generator $A+F$ of the evolution semigroup $\left\{T^{h} \mid h \geq 0\right\}$ is closed and densely defined in $L_{p}(\mathbf{X}), 1 \leq p<\infty$ or in $C_{0}(\mathbb{R}, \mathbf{X})$, respectively.

Proof. Since $f(t, x)$ is Lipschitz continuous with respect to $x$ uniformly in $t$ and $f(t, 0) \equiv 0$, the operator $F$ is continuous in the function spaces $L_{p}(\mathbf{X}), 1 \leq p<\infty$ and $C_{0}(\mathbb{R}, \mathbf{X})$. Thus, the assertions of the corollary follow from Proposition 2.

Now we suppose that all assumptions of Proposition 2 are satisfied and that $A+F$ generates a nonlinear semigroup in some sense (e.g. in the Crandall-Liggett sense [12]). Then the question arises of how to relate this semigroup to the associated evolution semigroup. In the general Banach space setting we need some additional conditions to see that they indeed coincide. In order to deal with those conditions we recall some notions which turn out to be useful later on. We first define

$$
[z, w]=\lim _{\lambda \rightarrow 0^{+}}(\|z+\lambda w\|-\|z\|) / \lambda
$$

and quote some important properties of this bracket $[\cdot, \cdot]$ from $[11$, p. 308]. 
Proposition 3. For $x, y, z \in \mathbf{X}$ and $\alpha, \beta \in \mathbb{R}$ we get the following properties:

i) $[\cdot, \cdot]: \mathbf{X} \times \mathbf{X} \rightarrow \mathbb{R}$ is upper-semicontinuous,

ii) $[\alpha x, \beta y]=|\beta|[x, y]$ if $\alpha \beta>0$,

iii) $[x, \alpha x+y]=\alpha\|x\|+[x, y]$,

iv) $|[x, y]| \leq\|y\|$ and $[0, y]=\|y\|$,

v) $-[x,-y] \leq[x, y]$,

vi) $[x, y+z] \leq[x, y]+[x, z]$

vii) $|[x, y]-[x, z]| \leq\|y-z\|$.

Definition 4. (see [11]) If $A$ is an operator in $\mathbf{X}$ and $\omega$ a real number, then $A+\omega I$ is called accretive if one (or all) of the following equivalent conditions hold:

i) $(1-\lambda \omega)\|x-y\| \leq\left\|x-y+\lambda\left(x^{\prime}-y^{\prime}\right)\right\|$ for all $x^{\prime} \in A x, y^{\prime} \in$ Ay and $\lambda \geq 0$.

ii) $\left[x-y, x^{\prime}-y^{\prime}\right] \geq-\omega\|x-y\|$ for all $x^{\prime} \in A x, y^{\prime} \in A y$.

iii) If $\lambda>0$ and $\lambda \omega<1$, then $(I+\lambda A)^{-1}$ is single-valued and has $(1-\lambda \omega)^{-1}$ as a Lipschitz constant.

Definition 5. A continuous function $f(t, x)$ is said to satisfy condition $H\left(C_{u}\right)$ or $H\left(C_{0}\right)$, respectively, if the mapping taking $v$ from $C_{u}(\mathbb{R}, \mathbf{X})$ or $C_{0}(\mathbf{X})$, respectively, into the function $f(\cdot, v(\cdot))$ is continuous.

Corollary 2. Under the assumptions of Proposition 2 the following is true:

i) Suppose that $-(A+F)$ is accretive and $R(I-\lambda(A+F))$ equals $L_{p}(\mathbf{X})$ or $C_{0}(\mathbf{X})$, where $A+F$ acts in $L_{p}(\mathbf{X})$ or in $C_{0}(\mathbf{X})$, respectively, for all sufficiently small positive $\lambda$. Then the zero solution of equation (1) is globally uniformly stable.

ii) Suppose that there exists a positive number $\alpha$ such that $\alpha I-(A+F)$ is accretive and $R\left(I-\lambda(\alpha I-(A+F))\right.$ equals $L_{p}(\mathbf{X})$ or $C_{0}(\mathbf{X})$ for all sufficiently small positive $\lambda$. Then the zero solution of equation (1) is globally exponentially stable.

Proof. Under the given assumptions the operator $A+F$ generates a semigroup of nonlinear operators on $L_{p}(\mathbf{X})$ or $C_{0}(\mathbf{X})$, respectively, in the CrandallLiggett sense [12]. In virtue of Corollary 1 we can apply [6, Corollary 4.3] to see that this semigroup coincides with the evolution semigroup associated with equation (1). Thus the assertion follows.

Remark. In Proposition 2 the perturbation $f(t, x)$ is assumed to be Lipschitz continuous (with Lipschitz constant $L$ ) with respect to $x$ uniformly in $t$ and $f(t, 0) \equiv 0$. If $\omega I-A$ is accretive, then $(L+\omega) I-(A+F)$ is $m$-accretive (see [48]).

Below we will weaken the conditions imposed on $f$ but then we have to restrict our considerations to a smaller class of well-posed equations of the form (4) which generate linear processes $\{U(t, s) \mid t \geq s\}$ such that $A$ is accretive. 
It turns out that suggested by the above results and by using the operator $A+F$ we can prove a version of Proposition 2 for a larger class of perturbations $f$ as well as the existence of mild solutions of equation (1). As we are concerned with the existence problem for solutions of equations of the form (1) we suppose that equation (1) satisfies the Uniqueness Condition for mild solutions, i.e. we suppose that for every fixed $s$ and $x$, if there exist two mild solutions $u_{1}(t)$ and $u_{2}(t)$ defined on some interval $[s, s+\delta), \delta>0$, then those two solutions coincide on this interval. In the appendix of this paper we describe sufficient conditions for this kind of uniqueness.

Theorem 1. Let the following conditions be satisfied:

i) The linear equation (4) is well-posed.

ii) Let $A$ denote the infinitesimal generator of the linear evolution semigroup associated with equation (4). Then $\alpha I-A$ is m-accretive.

iii) $f(t, x)$ satisfies condition $H\left(C_{0}\right)$ and $\beta I-F$ is accretive.

iv) Equation (1) satisfies the Uniqueness Condition on mild solutions.

Then equation (1) generates a continuous evolutionary process whose associated evolution semigroup is strongly continuous in $C_{0}(\mathbb{R}, \mathbf{X})$ and has $A+F$ as its infinitesimal generator with domain $D(A+F)=D(A) \subset C_{0}(\mathbb{R}, \mathbf{X})$. Furthermore, this evolution semigroup satisfies the estimate

$$
\left\|S^{h} v-S^{h} w\right\| \leq e^{(\alpha+\beta) h}\|v-w\| \quad \text { for all } v, w \in C_{0}(\mathbb{R}, \mathbf{X}), h \geq 0 .
$$

Proof. Under the assumptions of the theorem the autonomous equation

$$
\frac{d u}{d t}=(A+F) u, \quad t \geq 0
$$

generates a strongly continuous semigroup $\left\{S^{t} \mid t \geq 0\right\}$ in Webb's sense (see [48]), i.e. $S^{t} u$ is the unique continuous solution of the integral equation

$$
S^{t} u=T(t) u+\int_{0}^{t} T(t-\xi) F S^{\xi} u d \xi,
$$

where $T(t)$ is generated by the linear operator $A$. Furthermore,

$$
\left\|S^{t} u-S^{t} v\right\| \leq e^{(\alpha+\beta) t}\|u-v\| \quad \text { for all } t \geq 0, u, v \in C_{0}(\mathbb{R}, \mathbf{X}) .
$$

In view of (16) we have

$$
\begin{aligned}
\left(S^{t-s} v\right)(t) & =(T(t-s) v)(t)+\int_{0}^{t-s}\left(T(t-s-\xi) F S^{\xi} u\right)(t) d \xi \\
& =U(t, s) v(s)+\int_{0}^{t-s} U(t, \xi+s)\left(F S^{\xi} v\right)(s+\xi) d \xi \\
& =U(t, s) v(s)+\int_{s}^{t} U(t, \eta) f\left(\eta,\left(S^{\eta-s} v\right)(\eta)\right) d \eta
\end{aligned}
$$

Thus, in view of (17) we observe that for every $s \in \mathbb{R}$ and $x \in \mathbf{X}$ equation (5) has at least one continuous solution $X(t, s) x=\left(S^{t-s} v\right)(t)$, where $v$ is any element of $C_{0}(\mathbb{R}, \mathbf{X})$ such that $v(s)=x$. Now we are going to show that 
$X(t, s) x$ depends continuously on $(t, s, x)$. Indeed, suppose that $x, x^{\prime} \in \mathbf{X}$ and

$$
v(t)=\left\{\begin{array}{cl}
(1-|t|) x & \text { for }|t| \leq 1, \\
0 & \text { for }|t|>1,
\end{array} \quad v^{\prime}(t)=\left\{\begin{array}{cl}
(1-|t|) x^{\prime} & \text { for }|t| \leq 1 \\
0 & \text { for }|t|>1
\end{array}\right.\right.
$$

Then, since $\left(S^{t-s} v\right)(t)=X(t, s) v(s)$, we get

$$
\begin{aligned}
& \left\|X\left(t^{\prime}, s^{\prime}\right) v^{\prime}(s)-X(t, s) x\right\|=\left\|\left(S^{t^{\prime}-s^{\prime}} v^{\prime}\right)\left(t^{\prime}\right)-\left(S^{t-s} v\right)(t)\right\| \\
& \leq\left\|\left(S^{t^{\prime}-s^{\prime}} v^{\prime}\right)\left(t^{\prime}\right)-\left(S^{t-s} v\right)\left(t^{\prime}\right)\right\|+\left\|\left(S^{t-s} v\right)\left(t^{\prime}\right)-\left(S^{t-s} v\right)(t)\right\| .
\end{aligned}
$$

If $t, s, v$ are fixed, then $\lim _{t^{\prime} \rightarrow t}\left\|\left(S^{t-s} v\right)\left(t^{\prime}\right)-\left(S^{t-s} v\right)(t)\right\|=0$. On the other hand,

$$
\begin{aligned}
& \left\|\left(S^{t^{\prime}-s^{\prime}} v^{\prime}\right)\left(t^{\prime}\right)-\left(S^{t-s} v\right)\left(t^{\prime}\right)\right\| \leq \sup _{\xi}\left\|\left(S^{t^{\prime}-s^{\prime}} v^{\prime}\right)(\xi)-\left(S^{t-s} v\right)(\xi)\right\| \\
& =\left\|S^{t^{\prime}-s^{\prime}} v^{\prime}-S^{t-s} v\right\| \leq\left\|S^{t^{\prime}-s^{\prime}} v^{\prime}-S^{t^{\prime}-s^{\prime}} v\right\|+\left\|S^{t^{\prime}-s^{\prime}} v-S^{t-s} v\right\| .
\end{aligned}
$$

In view of the strong continuity of $S^{t}$ and the property

$$
\left\|S^{t^{\prime}-s^{\prime}} v^{\prime}-S^{t^{\prime}-s^{\prime}} v\right\| \leq e^{(\alpha+\beta)\left(t^{\prime}-s^{\prime}\right)}\left\|v^{\prime}-v\right\|
$$

we have

$$
\lim _{\left(t^{\prime}, s^{\prime}, v^{\prime}\right) \rightarrow(t, s, v)}\left\|\left(S^{t^{\prime}-s^{\prime}} v^{\prime}\right)\left(t^{\prime}\right)-\left(S^{t-s} v\right)(t)\right\|=0 .
$$

This shows that $X(t, s) x$ depends continuously on $(t, s, x)$. Finally, it is clear that

$$
\|X(t, s) x-X(t, s) y\| \leq e^{(\alpha+\beta)(t-s)}\|x-y\| \quad \text { for all } t \geq s, x, y \in \mathbf{X} .
$$

Thus we have proved that $\left\{S^{h} \mid h \geq 0\right\}$ is the evolution semigroup associated with the evolutionary process $\{X(t, s) \mid t \geq s\}$. This completes the proof of the theorem.

Now we apply Theorem 1 to investigate the stability of the mild solutions of equation (1).

Corollary 3. Let all assumptions of Theorem 1 be satisfied with $\alpha+\beta<0$. Then there exists a unique mild solution $x: \mathbb{R} \rightarrow C_{0}(\mathbb{R}, \mathbf{X})$ of equation (1) which is exponentially stable (among mild solutions).

Proof. In virtue of Theorem 1 we have

$$
\left\|S^{t} u-S^{t} v\right\| \leq e^{(\alpha+\beta) t}\|u-v\| \quad \text { for all } t \geq 0, u, v \in C_{0}(\mathbb{R}, \mathbf{X}) .
$$

Consequently, from the assumptions of the corollary it may be shown that the operators $S^{t}, t \geq 0$ have a unique common fixed point $v_{0} \in C_{0}(\mathbb{R}, \mathbf{X})$ which obviously represents a mild solution of equation (1). The stability of this solution follows immediately from the above estimate.

Remark. It may be noted that if the evolution semigroup $\left\{T^{h} \mid h \geq 0\right\}$ associated with the linear equation (4) is strongly continuous in $C_{u}(\mathbb{R}, \mathbf{X})$ and if $F$ acts on $C_{u}(\mathbb{R}, \mathbf{X})$, then Theorem 1 is still valid for $C_{u}(\mathbb{R}, \mathbf{X})$.

Theorem 2. Let the following conditions be satisfied: 
i) The linear equation (4) is well posed. Furthermore, the evolution semigroup associated with the linear process generated by equation (4) is strongly continuous in $C_{u}(\mathbb{R}, \mathbf{X})$.

ii) Let $A$ denote the infinitesimal generator of the above linear evolution semigroup. Then $\alpha I-A$ is m-accretive.

iii) $f$ satisfies condition $H\left(C_{u}\right)$ and $\beta I-F$ is accretive.

iv) Equation (1) satisfies the Uniqueness Condition on mild solutions.

Then equation (1) generates a continuous evolutionary process whose associated evolution semigroup is strongly continuous in $C_{u}(\mathbb{R}, \mathbf{X})$ and has $A+F$ as its infinitesimal generator with domain $D(A+F)=D(A) \subset C_{u}(\mathbb{R}, \mathbf{X})$. Furthermore,

$$
\left\|S^{h} v-S^{h} w\right\| \leq e^{(\alpha+\beta) h}\|v-w\| \quad \text { for all } v, w \in C_{u}(\mathbb{R}, \mathbf{X}), h \geq 0 .
$$

Proof. The theorem can be proved in the same manner as the previous one. So we omit the details.

In particular, if $A(t)=0$ for all $t$, then we get all assertions of Lemmas 1 and 2 of [33]. In this case $A=-d / d t$ with $D(A)=C_{u}^{1}(\mathbb{R}, \mathbf{X})$. Furthermore, Theorem 2 allows to improve substantially the results for nonlinear equations in [33].

Now we are going to discuss another application of the evolution semigroups $\left\{S^{h} \mid h \geq 0\right\}$ acting on $C_{u}(\mathbb{R}, \mathbf{X})$ to investigate the existence of periodic solutions of equation (1).

Definition 6. An evolutionary process $\{Z(t, s) \mid t \geq s\}$ is said to be $\tau$ periodic if

$$
Z(t+\tau, s+\tau)=Z(t, s) \text { for all } t \geq s .
$$

Theorem 3. Suppose the following conditions are satisfied:

i) The linear equation (4) is well-posed and it generates a $\tau$-periodic evolutionary process $\{U(t, s) \mid t \geq s\}$.

ii) $f(t, x)$ is $\tau$-periodic with respect to $t$ for every fixed $x$.

iii) Equation (1) generates an evolutionary process.

iv) $x_{0}(\cdot)$ is a unique fixed point of $S^{\tau}$ in a subset $\Omega$ of the space of all bounded functions $C_{b}(\mathbb{R}, \mathbf{X})$ on $\mathbb{R}$ which is invariant with respect to the semigroup $\left\{S^{h} \mid h \geq 0\right\}$ and the translation $S_{\tau}: x(\cdot) \rightarrow x(\cdot+\tau)$.

Then $x_{0}(t)$ is a $\tau$-periodic mild solution of equation (1).

Proof. We first prove that in $C_{b}(\mathbb{R}, \mathbf{X})$ one has $S^{\tau} S_{\tau}=S_{\tau} S^{\tau}$. In fact, by definition

$$
\begin{aligned}
X(t+\tau, s+\tau) x & =U(t+\tau, s+\tau) x+\int_{s+\tau}^{t+\tau} U(t+\tau, \xi) f(\xi, X(\xi, s+\tau) x) d \xi \\
& =U(t, s) x+\int_{s}^{t} U(t, \xi) f(\xi, X(\xi+\tau, s+\tau) x) d \xi
\end{aligned}
$$

Thus from the uniqueness we get

$$
X(t, s) x=X(t+\tau, s+\tau) x \quad \text { for all } t \geq s, x \in \mathbf{X} .
$$


This proves that $S^{\tau} S_{\tau}=S_{\tau} S^{\tau}$. Now since $S^{\tau}$ commutes with all other operators of the evolution semigroup $x_{0}$ has to be a common fixed point of $S^{h}$ and $S_{\tau}$. This implies that $x_{0}$ is a mild solution of equation (1) which is $\tau$-periodic. The proof of the theorem is complete.

Theorem 4. Let all assumptions of Theorem 2 be satisfied with $\alpha+\beta<0$. Furthermore, let the following conditions be fulfilled:

i) The linear equation (4) generates a $\tau$-periodic process and the evolution semigroup $\left\{T^{h} \mid h \geq 0\right\}$ associated with (4) is strongly continuous in $C_{u}(\mathbb{R}, \mathbf{X})$.

ii) $f(t, x)$ is $\tau$-periodic with respect to $t$ for every $x$.

Then equation (1) has a unique $\tau$-periodic mild solution which is globally exponentially stable.

Proof. This theorem is an immediate consequence of Theorems 2 and 3.

Another application of Theorem 3 is related to the concept of exponential dichotomy whose definition due to Henry [18] we recall next.

Definition 7. A linear evolutionary process $\{U(t, s) \mid t, s \in \mathbb{R}, t \geq s\}$ is said to have an exponential dichotomy if there exist positive constants $N, \alpha$ and projections $P(t), t \in \mathbb{R}$, bounded uniformly in $t$, i.e.

$$
\sup _{t \in \mathbb{R}}\|P(t)\|<\infty,
$$

such that the following three conditions hold:

i) $U(t, s) P(s)=P(t) U(t, s)$ for all $t \geq s$.

ii) For $t \geq s$ the restriction $\left.U(t, s)\right|_{\operatorname{Ker} P(s)}$ is an isomorphism from $\operatorname{Ker} P(s)$ onto Ker $P(t)$ and we define $U(s, t)$ as the inverse mapping from Ker $P(t)$ onto $\operatorname{Ker} P(s)$.

iii) The inequalities

$$
\begin{aligned}
\|U(t, s) P(s) x\| \leq N e^{-\alpha(t-s)}\|P(s) x\| \quad \text { for all } t \geq s, x \in \mathbf{X}, \\
\|U(t, s) Q(t) x\| \leq N e^{-\alpha(s-t)}\|Q(s) x\| \text { for all } s \geq t, x \in \mathbf{X},
\end{aligned}
$$

hold true where $X(t, s)$ for $s \geq t$ is defined in ii) and $Q(s):=I-P(s)$.

By abuse of terminology we say that a semigroup $\{T(t) \mid t \geq 0\}$ has an exponential dichotomy (or that it is hyperbolic) if the process $\{U(t, s) \mid t \geq s\}$ defined by

$$
U(t, s)=T(t-s) \quad \text { for all } t, s \in \mathbb{R}, t \geq s
$$

has an exponential dichotomy.

Proposition 4. Suppose that the following conditions are satisfied:

i) $A(t)$ is constant (with value a) for $t \geq 0$ and the semigroup $\{T(t) \mid t \geq 0\}$ has an exponential dichotomy,

ii) $f(t, x)$ is $\tau$-periodic with respect to $t$, continuous with respect to $(t, x)$ and Lipschitz continuous with respect to $x$ with Lipschitz constant $\delta$. 
Then for sufficiently small $\delta$ the equation

$$
\frac{d x}{d t}=a+f(t, x)
$$

has a unique $\tau$-periodic mild solution.

Proof. First notice that equation (19) generates an evolutionary process. Furthermore, observe that

$$
\begin{aligned}
\|X(t, s) x-X(t, s) y\| \leq & K e^{\omega(t-s)}\|x-y\| \\
& +\int_{s}^{t} K e^{\omega(t-\xi)} \delta\|X(\xi, s) x-X(\xi, s) y\| d \xi .
\end{aligned}
$$

Hence, using Gronwall's inequality we have

$$
\|X(t, s) x-X(t, s) y\| \leq K e^{(\omega+\delta K)(t-s)}\|x-y\| .
$$

Consequently, the evolution semigroup $\left\{S^{h} \mid h \geq 0\right\}$ associated with equation (19) acts on the space $C_{b}(\mathbb{R}, \mathbf{X})$ of bounded functions on $\mathbb{R}$. Now observe that

$$
\begin{aligned}
& \|(X(t, s) x-U(t, s) x)-(X(t, s) y-U(t, s) y)\| \leq \\
& \leq \delta \int_{s}^{t} K e^{\omega(t-\xi)}\|X(\xi, s) x-X(\xi, s) y\| d \xi \leq \\
& \leq \delta \int_{s}^{t} K e^{\omega(t-\xi)} K e^{(\omega+K L)(\xi-s)} d \xi\|x-y\|,
\end{aligned}
$$

where $U(t, s)=T(t-s)$ for all $t \geq s$. Thus, if $\delta$ is sufficiently small we can apply the Inverse Function Theorem for Lipschitz mappings (see e.g. [27], [34]) to conclude that $S^{\tau}$ has a unique fixed point. Now we are in a position to apply Theorem 3 to see that equation (19) has a unique $\tau$-periodic mild solution.

Remark. The results derived in this section can be generalized to hold for equations which are defined in closed subsets of the extended phase space. The corresponding proofs are based on the above approach applied to the results available for the autonomous case (see e.g. [24], [27]).

\section{Evolution SEMIGROUPS: UNSTABLE INTEGRAL MANIFOLDS AND INSTABILITY OF SOLUTIONS}

In this section we discuss the application of evolution semigroups to study the instability of solutions. To this end we prove the existence of unstable manifolds for semilinear equations whose linear parts have an exponential dichotomy. Since we deal with evolutionary processes rather than with concrete equations our results can be applied to a large class of evolution equations such as partial functional differential equations.

In this section we consider the (possibly nonlinear) perturbation $\{X(t, s) \mid$ $t \geq s\}$ of a given linear evolutionary process $\{U(t, s) \mid t \geq s\}$. By abuse of 
terminology we say that a function $x: \mathbb{R} \rightarrow \mathbf{X}$ is a solution of a given process $\{X(t, s) \mid t \geq s\}$ if

$$
X(t, s) x(s)=x(t) \text { for all } t \geq s .
$$

We then put

$$
\phi(t, s) x=X(t, s) x-U(t, s) x \quad \text { for all } t \geq s, x \in \mathbf{X} .
$$

For convenience, in this section we always assume that all the evolutionary processes $\{Z(t, s) \mid t \geq s\}$ under consideration have the property

$$
Z(t, s) 0=0 \text { for all } t \geq s .
$$

We say that the process $\{Z(t, s) \mid t \geq s\}$ has bounded growth if

$$
\|Z(t, s) x\| \leq M e^{\omega(t-s)}\|x\| \text { for all } t \geq s, x \in \mathbf{X}
$$

for some positive constants $\omega$ and $M$. Below we suppose that all evolutionary processes in consideration have bounded growth.

Definition 8. A set $M \subset \mathbb{R} \times \mathbf{X}$ is said to be an integral manifold of the evolutionary process $\{X(t, s) \mid t \geq s\}$ if for every $t \in \mathbb{R}$ the phase space $\mathbf{X}$ splits into a direct sum $\mathbf{X}=X_{t}^{1} \oplus X_{t}^{2}$ such that

$$
\inf _{t \in \mathbb{R}} \operatorname{Sn}\left(X_{t}^{1}, X_{t}^{2}\right) \stackrel{\text { def }}{=} \inf _{t \in \mathbb{R}} \inf _{x_{i} \in X_{t}^{i},\left\|x_{i}\right\|=1, i=1,2}\left\|x_{1}+x_{2}\right\|>0
$$

and if there exists a family of Lipschitz continuous mappings $g_{t}: X_{t}^{1} \rightarrow$ $X_{t}^{2}, t \in \mathbb{R}$, with Lipschitz constants independent of $t$ such that

$$
M=\left\{\left(t, x, g_{t}(x)\right) \in \mathbb{R} \times\left(X_{t}^{1} \oplus X_{t}^{2}\right) \mid t \in \mathbb{R}, x \in X_{t}^{1}\right\}
$$

and

$$
X(t, s)\left(g r\left(g_{s}\right)\right)=\operatorname{gr}\left(g_{t}\right) \text { for all } t \geq s,
$$

where $\operatorname{gr}\left(g_{s}\right)$ denotes the graph $\left\{(x, y) \in X_{s}^{1} \oplus X_{s}^{2} \mid y=g_{s}(x)\right\}$ of the mapping $g_{s}$. An integral manifold $M$ is said to be proper if the set $\{(t, 0,0) \in$ $\left.\mathbb{R} \times\left(X_{t}^{1} \oplus X_{t}^{2}\right) \mid t \in \mathbb{R}\right\}$ is contained in $M$.

We are going to show that every nonlinear process $\{Z(t, s) \mid t \geq s\}$ which is close enough to a linear process having an exponential dichotomy has an unstable integral manifold. The method of proof we use is the so-called graph transform (see e.g. [20], [34]).

Suppose that the linear process $\{U(t, s) \mid t \geq s\}$ has an exponential dichotomy with positive constants $K, \alpha$ and projections $P(t), t \in \mathbb{R}$. Since $\mathrm{P}(\mathrm{t})$ is bounded uniformly in $t$, i.e. $\sup _{t \in \mathbb{R}}\|P(t)\|<\infty$, from the well-known fact [13] that

$$
\frac{1}{\|P(t)\|} \leq \operatorname{Sn}(\operatorname{Im} P(t), \operatorname{Ker} P(t)) \leq \frac{2}{\|P(t)\|},
$$

it follows that

$$
\inf _{t \in \mathbb{R}} \operatorname{Sn}(\operatorname{Im} P(t), \operatorname{Ker} P(t))=\gamma>0
$$


From now on we use the notation $X_{t}^{1}=\operatorname{Im} P(t), X_{t}^{2}=\operatorname{Ker} P(t)$. Furthermore, for every fixed $r>0$ we denote by $B_{t}^{1}(r), B_{t}^{2}(r)$ and $B(r)$ the open balls of radius $r$ in the Banach spaces $X_{t}^{1}, X_{t}^{2}$ and $\mathbf{X}$, respectively. We have

$$
\|x\| \leq\|P(t) x\|+\|Q(t) x\| \leq\left(2 \sup _{t}\|P(t)\|+1\right)\|x\|,
$$

where $Q(t)=I-P(t)$. Thus we get for all $t \in \mathbb{R}$ and $x \in \mathbf{X}$

$$
\frac{1}{2}\|x\| \leq \max \{\|P(t) x\|,\|Q(t) x\|\} \leq\left(1+\sup _{t}\|P(t)\|\right)\|x\| .
$$

Below we shall assume that

$$
\|\phi(t, s) x-\phi(t, s) y\| \leq \epsilon e^{\mu(t-s)}\|x-y\| \quad \text { for all } t \geq s, x, y \in \mathbf{X},
$$

for some positive constants $\epsilon$ and $\mu$. Putting

$$
O_{\delta}=\left\{g_{t}: X_{t}^{2} \rightarrow X_{t}^{1} \mid g_{t}(0)=0, \operatorname{Lip}\left(g_{t}\right) \leq \delta, t \in \mathbb{R}\right\}
$$

we define in $O_{\delta}$ a distance

$$
d(g, h)=\sum_{k=1}^{\infty} \frac{1}{2^{k}} \sup _{t \in \mathbb{R},\|x\| \leq k}\left\|g_{t}(x)-h_{t}(x)\right\| .
$$

It is easily checked that $\left(O_{\delta}, d\right)$ is a complete metric space.

Proposition 5. Assume that the linear process $\{U(t, s) \mid t \geq s\}$ has an exponential dichotomy with constants $K, \alpha$ and projections $P(t), t \in \mathbb{R}$ and suppose $h_{0}$ is a given positive number. Then there exists a positive constant $\delta_{0}$ (depending only on $\{U(t, s) \mid t \geq s\}$ and $h_{0}$ ) such that for any $0<\delta<\delta_{0}$ the mapping $Q(t) U(t, s)\left(g_{s}(x), x\right)$ is a homeomorphism with respect to $x$ from $X_{s}^{2}$ onto $X_{t}^{2}$ for all $0 \leq t-s \leq h_{0}$.

Similarly, for $\delta<\delta_{0} / 2$ and $\epsilon<\delta_{0} e^{-\mu h_{0}} / 2$ the mapping $Q(t) X(t, s)$ $\circ\left(g_{s}(x), x\right)$ is a homeomorphism with respect to $x$ from $X_{s}^{2}$ onto $X_{t}^{2}$.

Proof. Consider the inclusion $i: x \longmapsto\left(g_{s}(x), x\right)$ and the mapping $Q(t) U(t, s) i$. Evidently, $Q(t) U(t, s) i$ is a linear homeomorphism from $X_{s}^{2}$ onto $X_{t}^{2}$. Let us define $\Gamma g_{s}$ as $\Gamma g_{s} x=\left(g_{s}(x), x\right)$. Applying the Inverse Function Theorem for Lipschitz continuous mappings (see e.g. [27], [34]) and putting

$$
\psi(t, s) x=Q(t) U(t, s) x-Q(t) U(t, s) \Gamma g_{s} x,
$$

we see that $\operatorname{Lip}(\psi) \leq \delta$, and if

$$
\delta \leq \frac{1}{K} \leq\left\|(Q(t) Y(t, s) i)^{-1}\right\|^{-1},
$$

then $Q(t) U(t, s) \Gamma g_{s}$ is a homeomorphism. Thus

$$
\delta_{0}=\frac{1}{K} \text {. }
$$

Similarly, if $\delta<\delta_{0} / 2$ and $\epsilon<\delta_{0} e^{-\mu h_{0}} / 2$, then $Q(t) U(t, s) \Gamma g_{s}$ is a homeomorphism. 
Proposition 6. Under the assumptions and notations of the previous proposition, if

$$
\delta\|Q(s)(x-y)\| \geq\|P(s)(x-y)\|
$$

then

$$
\delta^{\prime}\|Q(t)(X(t, s) x-X(t, s) y)\| \geq\|P(t)(X(t, s) x-X(t, s) y)\|,
$$

where

$$
\delta^{\prime}=\frac{\delta K e^{-\alpha(t-s)}+2 \epsilon e^{\mu(t-s)}}{(1 / K) e^{\alpha(t-s)}-2 \epsilon e^{\mu(t-s)}} .
$$

Proof. Putting $f=X(t, s), S=U(t, s)$ and $\phi=\phi(t, s)$ for simplicity we get

$$
\|Q(t) f(x)-Q(t) f(y)\| \geq\|(Q(t) S x-Q(t) S x)+(\phi(x)-\phi(y))\| .
$$

Since $Q(t) U(t, s)=Q(t) U(t, s) Q(s)$ we have

$$
\|Q(t) f(x)-Q(t) f(y)\| \geq(1 / K) e^{\alpha(t-s)}\|Q(s)(x-y)\|-\epsilon e^{\mu(t-s)}\|x-y\| .
$$

Taking into account (25) and (27), for sufficiently small $\delta\left(\delta<\delta_{0}\right)$ we get

$$
\|Q(t) f(x)-Q(t) f(y)\| \geq\left[(1 / K) e^{\alpha(t-s)}-2 \epsilon e^{\mu(t-s)}\right]\|Q(s)(x-y)\| .
$$

On the other hand, we have

$$
\begin{aligned}
\|P(t) f(x)-P(t) f(y)\| & =\|(P(t) S x-P(t) S y)+(\phi(x)-\phi(y))\| \\
& \leq K e^{-\alpha(t-s)}\|P(s)(x-y)\|+\epsilon e^{\mu(t-s)}\|x-y\| .
\end{aligned}
$$

According to (25) and (27) we have

$$
\|P(t) f(x)-P(t) f(y)\| \leq\left[\delta K e^{-\alpha(t-s)}+2 \epsilon e^{\mu(t-s)}\|Q(s)(x-y)\| .\right.
$$

Thus, from (30) and (31) it follows that

$$
\|P(t) f(x)-P(t) f(y)\| \leq \delta^{\prime}\|Q(t) f(x)-Q(t) f(y)\| .
$$

This completes the proof of the proposition.

From Propositions 5 and 6 we see that for a given positive $h_{0}$ if $\delta<\delta_{0} / 2$ and $\epsilon<\left(\delta_{0} e^{-\mu h_{0}}\right) / 2$, then $S^{h}$ is well defined as a mapping from $O_{\delta}$ to $O_{\delta^{\prime}}$ for $0<h<h_{0}$.

Now we choose $k \in \mathbb{N}$ such that

$$
K e^{-\alpha k}=q<\frac{1}{2}
$$

and then $h_{0}=2 k$. Thus, for $\delta<\delta_{0} / 2=1 /(2 K)$ and

$$
0<\epsilon<\min \left\{\frac{e^{-2 \mu k}}{2 K}, \frac{\delta\left(q^{-1}-q\right)}{2(1+\delta)} e^{-2 \mu k}\right\}
$$

$S^{k}$ maps $O_{\delta}$ into itself by the formula

$$
g r\left(\left(S^{k} g\right)_{t}\right)=X(t, t-k)\left(g r\left(g_{t-k}\right)\right) \quad \text { for all } g \in O_{\delta} .
$$


Proposition 7. Under the above assumptions on $\{U(t, s) \mid t \geq s\}$ and $\{X(t, s) \mid t \geq s\}$, for sufficiently small $\epsilon, S^{k}$ is a contraction mapping in $O_{\delta}$.

Proof. It is sufficient to show that for some $0<q^{\prime}<1$ the estimate

$$
\begin{aligned}
& \left\|P(t) X(t, t-k) x-\left(S^{k} g\right)_{t}(Q(t) X(t, t-k) x)\right\| \\
& \left.\leq q^{\prime} \| P(t-k) x-g_{t-k} Q(t-k) x\right) \|
\end{aligned}
$$

is true for every $g \in O_{\delta}$ and $x \in \mathbf{X}$. In fact, suppose that $h \in O_{\delta}$, substituting $x$ by $\left(h_{t-k}(Q(t-k) x), Q(t-k) x\right)$ into (33) we get

$$
\begin{aligned}
& \left\|\left(S^{k} h\right)_{t}(Q(t) X(t, t-k) x)-\left(S^{k} g\right)_{t}(Q(t) X(t, t-k) x)\right\| \\
& \leq q^{\prime}\left\|h_{t-k}(Q(t-k) x)-g_{t-k}(Q(t-k) x)\right\|
\end{aligned}
$$

for all $x \in \mathbf{X}$ and $t \in \mathbb{R}$. Put $y=Q(t-k) x$. Then $[Q(t) X(t, t-k) Q(t-$ $k)]^{-1}(\{\|z\| \leq r\})$ is contained in $\{\|y\| \leq r\}$. Thus, for every $n \in \mathbb{N}$

$$
\sup _{t \in \mathbb{R},\|y\| \leq n}\left\|\left(S^{k} h\right)_{t}(y)-\left(S^{k} g\right)_{t}(y)\right\| \leq q^{\prime} \sup _{t \in \mathbb{R},\|y\| \leq n}\left\|h_{t}(y)-g_{t}(y)\right\|
$$

Hence, for sufficiently small $\epsilon$ (such that $\left.q^{\prime}<1\right), S^{k}$ is a contraction mapping.

Now we prove that (33) holds. For simplicity of notation put $f=X(t, t-$ $k), S=U(t, t-k)$ and $\phi=X(t, t-k)-U(t, t-k)$. We then have

$$
\begin{aligned}
& \left\|Q(t) f(x)-Q(t) f\left(g_{t-k}(Q(t-k) x)+Q(t-k) x\right)\right\| \\
& \leq\left\|Q(t) \phi(x)-Q(t) \phi\left(g_{t-k}(Q(t-k) x)+Q(t-k) x\right)\right\| \\
& \quad+\left\|Q(t) S(x)-Q(t) S\left(g_{t-k}(Q(t-k) x)+Q(t-k) x\right)\right\| \\
& \leq \sup _{t}\|Q(t)\| \epsilon e^{\mu k}\left\|P(t-k) x-g_{t-k}(Q(t-k) x)\right\| .
\end{aligned}
$$

On the other hand, we have

$$
\begin{aligned}
& \left\|P(t) f(x)-P(t) f\left(g_{t-k}(Q(t-k) x)+Q(t-k) x\right)\right\| \\
& \leq\left\|P(t)\left(\phi(x)-\phi\left(g_{t-k}(Q(t-k) x)+Q(t-k) x\right)\right)\right\| \\
& \quad+\left\|P(t)\left(S(x)-S\left(g_{t-k}(Q(t-k) x)+Q(t-k) x\right)\right)\right\| \\
& \leq \sup _{t}\|P(t)\| \epsilon e^{\mu k}\left\|P(t-k) x-g_{t-k}(Q(t-k) x)\right\| \\
& \quad+K e^{-\alpha k}\left\|P(t-k) x-g_{t-k}(Q(t-k) x)\right\| \\
& =\left(q+\sup _{t}\|P(t)\| \epsilon e^{\mu k}\right)\left\|P(t-k) x-g_{t-k}(Q(t-k) x)\right\| .
\end{aligned}
$$


Note that $\left(S^{k} g\right)_{t}(Q(t) Z(t, t-k) x)=P(t) f\left(g_{t-k}(Q(t-k) x)+Q(t-k) x\right)$. Now, combining (35) and (36), we get

$$
\begin{aligned}
\| & P(t) X(t, t-k) x-\left(S^{k} g\right)_{t}(Q(t) X(t, t-k) x) \| \\
\leq & \| P(t) f(x) \\
& -\left(S^{k} g\right)_{t}\left(Q(t) X(t, t-k)\left(g_{t-k}(Q(t-k) x)+Q(t-k) x\right)\right) \| \\
& +\|\left(S^{k} g\right)_{t}\left(Q(t) X(t, t-k)\left(g_{t-k}(Q(t-k) x)+Q(t-k) x\right)\right) \\
& -\left(S^{k} g\right)_{t}(Q(t) X(t, t-k) x) \| \\
\leq & \left\|P(t) f(x)-P(t) f\left(g_{t-k}(Q(t-k) x)+Q(t-k) x\right)\right\| \\
& +\| P(t) f\left(g_{t-k}(Q(t-k) x)\right. \\
& +Q(t-k) x)-\left(S^{k} g\right)_{t}(Q(t) Z(t, t-k) x \| \\
\leq & \left(q+\sup _{t}\|P(t)\| \epsilon e^{\mu k}+\delta\right)\left\|P(t-k) x-g_{t-k}(Q(t-k) x)\right\| .
\end{aligned}
$$

Thus $q^{\prime}=q+\delta+\sup _{t}\|P(t)\| \epsilon e^{\mu k}$ is less than 1 if $\epsilon$ and $\delta$ are sufficiently small. This proves the assertion of the proposition.

Supposing that $g^{\prime}$ is the fixed point of $S^{k}$ in $O_{\delta}$, we next prove that $g^{\prime}$ is the fixed point of $S^{h}$ for all $h \geq 0$ in some sense.

Theorem 5. Under the assumptions of Propositions 6 and 7 there exists a so-called unstable integral manifold (which is proper and Lipschitz continuous) for the nonlinear evolutionary process $\{X(t, s) \mid t \geq s\}$.

Proof. We only need to prove that $M=\left\{g r\left(g_{t}^{\prime}\right) \mid t \in \mathbb{R}\right\}$ is left invariant by the process $\{X(t, s) \mid t \geq s\}$, i.e. that

$$
\operatorname{gr}\left(g_{t}^{\prime}\right)=X(t, s)\left(\operatorname{gr}\left(g_{s}^{\prime}\right)\right) \text { for all } t \geq s .
$$

To this end we consider the action of $S^{h}, 0 \leq h \leq 2 k$ on $O_{\delta}$ for sufficiently small $\delta$ and $\epsilon$, by the formula

$$
\operatorname{gr}\left(g_{t}\right)=X(t, t-h)\left(\operatorname{gr}\left(g_{t-h}\right)\right),
$$

where $g=\left\{g_{t} \mid t \in \mathbb{R}\right\} \in O_{\delta}$. According to Proposition 6 we can choose $\delta$ and $\epsilon$ sufficiently small so that

$$
\sup _{0 \leq t-s \leq 2 k} \delta^{\prime}<\frac{\delta_{0}}{4}=\delta_{1}
$$

where $\delta^{\prime}$ is defined by $(29), \delta_{0}=K e^{-2 \alpha k}$. Thus $S^{h}$ is a mapping from $O_{\delta}$ to $O_{\delta^{\prime}}$. Suppose that $\epsilon$ and $\delta$ are chosen such that (32) holds. Then for any $\xi \in[0, k)$ we consider the mappings $S^{k+\xi}: O_{\delta} \rightarrow O_{\delta_{1}}$ and $S^{h}: O_{\theta} \rightarrow O_{\theta}, \delta \leq$ $\theta \leq \delta_{1}$. We then get

$$
S^{k+\xi}=S^{\xi} \cdot S^{k}\left(: O_{\delta} \rightarrow O_{\delta_{1}}\right)=S^{k} \cdot S^{\xi}\left(O_{\delta} \rightarrow O_{\delta_{1}}\right) .
$$

From Proposition 7 we have

$$
S^{\xi} \cdot S^{k} g^{\prime}=S^{\xi} g^{\prime} \text { and } S^{\xi} \cdot S^{k} g^{\prime}=S^{k} \cdot S^{\xi} g^{\prime}=S^{\xi} g^{\prime} .
$$


From the uniqueness of the fixed point of $S^{k}$ it follows that $S^{\xi} g^{\prime}=g^{\prime}$ where $g^{\prime}$ is the fixed point of $S^{k}$ acting on $O_{\delta}$. This proves the theorem.

Remark. It is apparent that $\operatorname{Lip}\left(g^{\prime}{ }_{t}\right) \rightarrow 0$ as $\epsilon \rightarrow 0$, where $g^{\prime}{ }_{t}, t \in \mathbb{R}$ is determined in the proof of Theorem 5 .

Combining the proofs of Propositions 6 and 7 with Theorem 5 we can deduce the following local version of Theorem 5 :

Theorem 6. Assume that $\{U(t, s) \mid t \geq s\}$ is as in Theorem 5 and that $\{X(t, s) \mid t \geq s\}$ is defined in the open ball $\{x \in \mathbf{X} \mid\|x\|<2 r\}$. Furthermore, suppose that (26) holds for all $x$ and $y$ in this ball. Then for sufficiently small $\epsilon$ there exists a "local" unstable integral manifold which is represented by $g=\left\{g_{t}: B_{t}^{2}\left(r_{t}\right) \rightarrow B_{t}^{1}\left(r_{t}\right)\right\}, \operatorname{Lip}\left(g_{t}\right)<\delta=\delta(\epsilon), \inf _{t} r_{t}>0$ such that

$$
\operatorname{gr}\left(g_{t}\right)=X(t, s)\left(g r\left(g_{s}\right)\right) \cap B\left(r_{s}\right) \quad \text { for all } t \geq s
$$

where $B(r)$ denotes the ball $\{x \in \mathbf{X} \mid \max \{\|P(t) x\|,\|Q(t) x\|\}<r\}$.

Proof. We can define a function $\rho: \mathbf{X} \rightarrow[0,1]$ with the property

$$
\rho(x)= \begin{cases}1 & \text { for }\|x\| \leq r \\ 0 & \text { for }\|x\| \geq 1.5 r\end{cases}
$$

with Lipschitz constant $L$. We then define

$$
X^{\prime}(t, s) x=\rho(x) X(t, s) x \quad \text { for all } x \in \mathbf{X} .
$$

Now, in order to complete the proof it suffices to apply Theorem 5 to $\left\{X^{\prime}(t, s) \mid t \geq s\right\}$.

Next we are going to apply the above results to investigate the asymptotic behaviour of the process $\{X(t, s) \mid t \geq s\}$ around the "zero solution".

Proposition 8. Under the assumptions of Theorem 5 we get the limiting relation

$$
\lim _{t \rightarrow \infty} d\left(Z(t, s) x, M_{t}\right)=0
$$

where $M_{t}=\operatorname{gr}\left(g_{t}\right)$ and $d\left(y, M_{t}\right)=\inf _{z \in M_{t}}\|y-z\|$.

Proof. From (33) it follows that

$$
d\left(X(t, t-k) x, M_{t}\right) \leq q^{\prime} d\left(x, M_{t-k}\right) \quad \text { for all } t \in \mathbb{R}, x \in \mathbf{X} .
$$

Thus we have

$$
\lim _{n \rightarrow \infty} d\left(Z(s+n k, s) x, M_{s+k}\right)=0 .
$$

From the bounded growth of $\{X(t, s) \mid t \geq s\}$ we get the claimed relation (39).

Below we shall consider the case where the linear process $\{U(t, s) \mid t \geq s\}$ satisfies a condition more general than that of an exponential dichotomy.

Definition 9. A linear process $\{U(t, s) \mid t \geq s\}$ with bounded growth is said to satisfy condition $H$ if there exist positive constants $K, \alpha, \beta$ with $\alpha>\beta$ and nontrivial projections $P(t), t \in \mathbb{R}$ which are bounded uniformly in $t$ such that the following three conditions are met: 
i) $P(t) U(t, s)=U(t, s) P(s)$ for all $t \geq s$,

ii) The restriction $\left.U(t, s)\right|_{\operatorname{Ker} P(s)}$ is an isomorphism from $\operatorname{Ker} P(s)$ onto $\operatorname{Ker} P(t)$ (whose inverse is denoted by $U(s, t)$ for $s \leq t$ ).

iii) With $Q(s)=I-P(s)$ we have

$$
\begin{aligned}
& \|U(t, s) P(s) x\| \leq K e^{-\alpha(t-s)}\|P(s) x\| \text { for all } t \geq s, x \in \mathbf{X}, \\
& \|U(t, s) Q(s) x\| \geq K^{-1} e^{-\beta(t-s)}\|Q(s) x\| \text { for all } t \geq s, x \in \mathbf{X} .
\end{aligned}
$$

Examples. It is apparent that every linear process with an exponential dichotomy satisfies condition $H$. More generally, one can show that a linear process $\{U(t, s) \mid t \geq s\}$ having bounded growth satisfies condition $H$ if and only if (see e.g. [25]) there exists an $r \in(0,1)$ such that the circle with radius $r$ belongs to the resolvent set $\rho(T(1))$ and that $\sigma(T(1)) \cap\{z \in \mathbb{C} \mid$ $|z|<r\} \neq \emptyset$, where $\{T(t) \mid t \geq 0\}$ is the evolution semigroup associated with $\{U(t, s) \mid t \geq s\}$ in $L_{p}$.

It is easy to see that if $\{U(t, s) \mid t \geq s\}$ satisfies condition $H$, then $\left\{U^{*}(t, s) \mid t \geq s\right\}$ defined as

$$
U^{*}(t, s) x=e^{\gamma(t-s)} U(t, s) x \quad \text { for all } x \in \mathbf{X}
$$

where $\gamma=(\alpha-\beta) / 2$ has an exponential dichotomy with constants $K,(\alpha-$ $\beta) / 2$ and the same projection $P(t), t \in \mathbb{R}$ as $\{U(t, s) \mid t \geq s\}$. Consider the "change of variables" for the nonlinear process $\{X(t, s) \mid t \geq s\}$ as follows: instead of $\{X(t, s) \mid t \geq s\}$ we consider the process $\left\{X^{*}(t, s) \mid t \geq s\right\}$ defined as

$$
X^{*}(t, s) x=e^{\gamma t} X(t, s)\left(e^{-\gamma s} x\right) \quad \text { for all } t \geq s, x \in \mathbf{X} .
$$

Observe that the process $\left\{X^{*}(t, s) \mid t \geq s\right\}$ is nonlinear as well. Furthermore, if the process

$$
\phi(t, s) x=X(t, s) x-U(t, s) x
$$

satisfies (26), then denoting

$$
\phi^{*}(t, s)=X^{*}(t, s) x-U^{*}(t, s) x
$$

we have

$$
\begin{aligned}
\| \phi^{*}(t, s) x & -\phi^{*}(t, s) y \| \\
& \leq e^{\gamma t}\left\|\phi(t, s)\left(e^{\gamma s} x\right)-\phi^{*}(t, s)\left(e^{\gamma s} y\right)\right\| \\
& \leq e^{\gamma t} \epsilon e^{\mu(t-s)} e^{\gamma s}\|x-y\| \\
& =\epsilon e^{(\gamma+\mu)(t-s)}\|x-y\| .
\end{aligned}
$$

Now we are in a position to apply Theorem 5 to the processes $\left\{U^{*}(t, s) \mid t \geq\right.$ $s\}$ and $\left\{X^{*}(t, s) \mid t \geq s\right\}$. It follows that for sufficiently small $\epsilon$ there exists a $g \in O_{\delta}$ (where $\delta=\delta(\epsilon)$ and $\lim _{\epsilon \rightarrow 0} \delta(\epsilon)=0$ ) such that $M=\left\{M_{t}=\right.$ $\left.\operatorname{gr}\left(g_{t}\right) \mid t \in \mathbb{R}\right\}$ is an integral manifold of $\left\{Z^{*}(t, s) \mid t \geq s\right\}$. Let us define $g_{t}^{*}(x)=e^{-\gamma t} g_{t}\left(e^{\gamma} t x\right)$. Obviously, we then get $\operatorname{gr}\left(g_{t}^{*}\right)=e^{-\gamma t} g r\left(g_{t}\right)$. Since $g=\left\{g_{t} \mid t \in \mathbb{R}\right\}$ satisfies

$$
\operatorname{gr}\left(g_{t}\right)=X^{*}(t, s)\left(\operatorname{gr}\left(g_{s}\right)\right) \quad \text { for all } t \geq s
$$


we get

$$
e^{\gamma t} g r\left(g_{t}^{*}\right)=e^{\gamma t} Z(t, s)\left(e^{-\gamma s} e^{\gamma s} g r\left(g_{s}^{*}\right)\right)
$$

This shows that the set $N=\left\{N_{t} \mid t \in \mathbb{R}\right\}$, where $N_{t}=\operatorname{gr}\left(g^{*}\right), t \in \mathbb{R}$ is an integral manifold of the process $\{X(t, s) \mid t \geq s\}$.

Now we are going to apply the above result to investigate the instability of solutions.

Theorem 7. (Reduction Principle for Stability of Evolutionary Processes.) Assume that the linear process $\{U(t, s) \mid t \geq s\}$ satisfies condition $H$. In addition assume that for the nonlinear process $\{X(t, s) \mid t \geq s\}$ the condition

$$
\operatorname{Lip}(X(t, s) x-U(t, s) x) \leq \epsilon e^{\mu(t-s)} \quad \text { for all } t \geq s
$$

holds for some positive $\mu$ and $\epsilon$.

Then for sufficiently small $\epsilon>0$ there exists an integral manifold $M=$ $\left\{M_{t} \mid t \in \mathbb{R}\right\}$ of $\{X(t, s) \mid t \geq s\}$ such that the zero solution of $\{X(t, s) \mid t \geq$ $s\}$ is stable if and only if for every $\epsilon^{*}>0$ and $s \in \mathbb{R}$ there exists a $\delta=$ $\delta\left(\epsilon^{*}, s\right)>0$ such that $\|X(t, s) x\|<\epsilon^{*}$ for all $t \geq s$ if $x \in M_{s}$ and $\|x\| \leq \delta$.

Similarly, the asymptotic, uniform and exponential stability of the zero solution of $\{X(t, s) \mid t \geq s\}$ are equivalent to the respective stability type of $\left\{\left.X(t, s)\right|_{M_{s}} \mid t \geq s\right\}$.

Proof. From (33) we can easily prove the assertions of the theorem. Thus the proof is similar to that of Proposition 8.

Remark. In the case where for every pair $(t, s)$ the operator $U(t, s)$ is compact we observe that codim $\operatorname{Im} P(t)<\infty$. Thus the integral manifold $M$ in Theorem 6 is of finite dimension, i.e. $\operatorname{dim} D\left(g_{t}\right)<\infty$ for all $t \in \mathbb{R}$.

Theorem 8. (Linearized Instability Theorem) Under the assumptions of Theorem 5, if the projections $P(t), t \in \mathbb{R}$ are non-trivial, i.e. $P(t) \neq I$ and $P(t) \neq 0$ for all $t$, then for sufficiently small $\epsilon$ the zero solution of $\{X(t, s) \mid t \geq s\}$ is unstable.

Proof . It is sufficient to prove that $X\left(s+k_{n}, s\right) x$ tends to $\infty$ as $k_{n} \rightarrow \infty$ for every $x \neq 0$ in $M_{s}$, where $M=\left\{M_{t} \mid t \in \mathbb{R}\right\}$ is the integral manifold provided by Theorem 5. For sufficiently small $\epsilon$ we have

$$
M_{t}=\operatorname{gr}\left(g_{t}\right), \quad \lim _{\epsilon \rightarrow 0} \delta(\epsilon)=0
$$


where $\operatorname{Lip}\left(g_{t}\right) \leq \delta=\delta(\epsilon)$. Thus we can assume that $\delta<1 / 2$. We have

$$
\begin{aligned}
\| X(t, s) & x \| \\
& =\|P(t) X(t, s) x+Q(t) X(t, s) x\| \\
& =\left\|g_{t}(Q(t) X(t, s) x)+Q(t) X(t, s) x\right\| \\
& \geq(1-\delta)\|Q(t) X(t, s) x\| \\
& \geq \frac{1}{2}\left\|Q(t) X(t, s)\left(g_{s}(Q(s) x)+Q(s) x\right)\right\| \\
& =\frac{1-\delta}{2}\|Q(t) X(t, s) Q(s) x\| \\
& \geq \frac{1}{4}\|Q(t) X(t, s) Q(s) x\| \\
& \geq \frac{1}{4}\left(\|Q(t) U(t, s) Q(s) x\|-\frac{1}{4} \sup _{t}\|Q(t)\| \operatorname{Lip}(\phi(t, s))\|Q(s) x\|\right) \\
& \geq \frac{1}{4}\left[\frac{1}{K} e^{\alpha(t-s)}-\sup _{t}\|Q(t)\| \epsilon e^{\mu(t-s)}\right]\|Q(s) x\| .
\end{aligned}
$$

Hence, if we fix $t-s=k_{0}$, where $k_{0}$ is chosen such that $(1 / K) e^{\alpha(t-s)}>8$, then for sufficiently small $\epsilon$ we have

$$
\left\|Q\left(k_{0}+s\right) X\left(k_{0}+s, s\right) Q(s) x\right\| \geq p\|Q(s) x\|,
$$

where $p>1$. Since $x \in \operatorname{gr}\left(g_{s}\right)$ we can apply (44) repeatedly to get

$$
\left\|Q\left(n k_{0}+s\right) X\left(n k_{0}+s, s\right) Q(s) x\right\| \geq p^{n}\|Q(s) x\| .
$$

Since $x \neq 0, x \in \operatorname{gr}\left(g_{s}\right)$ we have $\|Q(s) x\| \neq 0$. Now from (43) we observe that

$$
\lim _{n \rightarrow \infty}\left\|X\left(n k_{0}+s, s\right) x\right\|=\infty .
$$

This completes the proof of the theorem.

\section{An APPlicAtion to PARTial FUnCtional DIFFERENTIAL EQUATIONS}

Since the coefficient-operators $A(t)$ in the equations considered above are not assumed to be bounded the results of the previous sections have applications in the theory of partial differential equations. For a standard procedure of such an application we refer to [3], [37], [38]. On the other hand, taking into account that in Section 3 we deal with evolutionary processes rather than with concrete evolution equations, we will consider an application of the results of Section 3 to study the asymptotic behavior of solutions of a class of partial functional differential equations (for a standard procedure see [47]). In a forthcoming paper we shall deal with the evolution semigroups associated with this kind of equations in the context of the theory of strongly continuous semigroups of operators.

In the sequel we will use the following terminology. By $C=C([-r, 0], \mathbf{X})$, $r>0$, we denote the Banach space of continuous $\mathbf{X}$-valued functions on $[-r, 0]$ equipped with the supremum norm. If $u$ is a continuous function 
from $[a-r, b]$ to $\mathbf{X}$ and $t \in[a, b]$, then $u_{t}$ denotes the element of $C$ given by $u_{t}(\theta)=u(t+\theta)$ for $-r \leq \theta \leq 0$.

For the reader's convenience the following result is quoted from [47]:

Proposition 9. Suppose $F:[a, b] \times C \rightarrow \mathbf{X}$ is continuous and satisfies

$$
\|F(t, \phi)-F(t, \psi)\|_{\mathbf{X}} \leq L\|\psi-\phi\|_{C} \quad \text { for all } t \in[a, b], \phi, \psi \in C,
$$

where $L$ is a positive constant. Furthermore let $\{T(t) \mid t \geq 0\}$ be a strongly continuous semigroup of linear operators acting on $\mathbf{X}$. Then for every $\phi \in C$ there exists a unique continuous function $u:[a-r, b] \rightarrow \mathbf{X}$ which solves the initial value problem

$$
\left\{\begin{aligned}
u(t) & =T(t-a) \phi(0)+\int_{a}^{t} T(t-s) F\left(s, u_{s}\right) d s \quad \text { for all } t \in[a, b], \\
u_{\alpha} & =\phi .
\end{aligned}\right.
$$

Proposition 10. Let the assumptions of Proposition 9 be satisfied for all $t \in[a, b]$ and suppose $L$ is independent of $a$ and $b$. Then equation (47) provides a (nonlinear) evolutionary process $\{X(t, s) \mid t \geq s\}$ on $C$. If in addition $F(t, 0)=0$ and $\|T(t)\| \leq e^{\mu t}$ for all $t \in \mathbb{R}$ as well as $L<1$, then for $\{X(t, s) \mid t \geq s\}$ the following holds:

$$
\|\Delta \phi-\Delta \psi\|_{C} \leq \epsilon e^{\omega^{\prime}(t-s)}\|\phi-\psi\|_{C} \quad \text { for all } t \geq s, \phi, \psi \in C,
$$

where

$$
\begin{aligned}
\epsilon & =L e^{2|\mu| r}, \quad \omega^{\prime}=1+|\mu|+e^{|\mu| r} \\
\Delta \phi & =X(t, s) \phi-U(t, s) \phi, \\
(U(t, s) \phi)(\theta) & =T(t+\theta-s) \phi(0) \quad \text { for all } t \geq s,-r \leq \theta \leq 0 .
\end{aligned}
$$

Proof. Suppose that $u(t)$ is the solution of equation (47). Then we put $X(t, a) \phi=u_{t}$. Now we show that $\{X(t, s) \mid t \geq s\}$ is an evolutionary process. To this end, it is sufficient to prove that

$$
X(t, s) \cdot X(s, \tau)=X(t, \tau) \text { for all } t \geq s \geq \tau .
$$

In virtue of Proposition 9 , if $u(t)$ denotes the solution of the equation

$$
\left\{\begin{aligned}
u(t) & =T(t-s)[X(s, \tau) \phi](0)+\int_{s}^{t} T(t-\xi) F\left(\xi, u_{\xi}\right) d \xi \quad \text { for all } t \geq s, \\
u_{s} & =X(s, \tau) \phi
\end{aligned}\right.
$$

then we have

$$
\begin{aligned}
u^{*}(t)= & T(t-s)\left[T(s-\tau) \phi(0)+\int_{\tau}^{s} T(s-\xi) F(\xi, X(\xi, \tau) \phi) d \xi\right] \\
& +\int_{s}^{t} T(t-\xi) F\left(\xi, u_{\xi}\right) d \xi \\
= & T(t-\tau) \phi(0)+\int_{\tau}^{t} T(t-\xi) F\left(\xi, u_{\xi}^{*}\right) d \xi,
\end{aligned}
$$

where

$$
u^{*}(\xi)=\left\{\begin{array}{cl}
u(\xi) & \text { for } \xi \geq s \\
X(\xi, \tau) \phi & \text { for } \tau \leq \xi \leq s
\end{array}\right.
$$


From the uniqueness of solutions it follows that $u^{*}(\xi)$ is the solution of the equation

$$
\left\{\begin{aligned}
u^{*}(t) & =T(t-\tau) \phi(0)+\int_{\tau}^{t} T(t-\xi) F\left(\xi, u^{*}(\xi) d \xi,\right. \\
u_{\tau}^{*} & =\phi .
\end{aligned}\right.
$$

Thus, by definition we have

$$
X(t, \tau) \phi=X(t, s)[X(s, \tau) \phi] \quad \text { for all } \phi \in C .
$$

This shows that $\{X(t, s) \mid t \geq s\}$ is indeed a (nonlinear) evolutionary process.

By assumptions there exists a positive constant $\mu$ such that

$$
\|T(t)\| \leq e^{\mu t} \quad \text { for all } t \geq 0 .
$$

Hence, since $F(t, 0) \equiv 0$, for all $t \geq s$ and $\phi \in C$ we have (see [47])

$$
\|X(t, s) \phi\| \leq\left\{\begin{array}{cl}
e^{(\mu+L)(t-s)}\|\phi\| & \text { for } \mu \geq 0 \\
e^{-\mu r} e^{\left(\mu+L e^{-\mu r}\right)(t-s)}\|\phi\| & \text { for } \mu<0 .
\end{array}\right.
$$

By definition we have

$$
\begin{aligned}
\| \Delta \phi & -\Delta \psi \| \\
& \leq \sup _{-r \leq \theta \leq 0} \int_{s}^{t+\theta} L\|T(t+\theta-\xi)\|\|X(\xi, s) \phi-X(\xi, s) \psi\| d \xi .
\end{aligned}
$$

On the other hand, we get

$$
\begin{aligned}
\|X(t, s) \phi-X(t, s) \psi\|_{C} \leq & e^{|\mu| r} e^{\mu(t-s)}\|\phi-\psi\|_{C}+ \\
& +\int_{s}^{t} e^{|\mu| r} e^{|\mu|(t-\xi)} L\|X(\xi, s) \phi-X(\xi, s) \psi\|_{C} d \xi .
\end{aligned}
$$

Putting

$$
g(t)=e^{-|\mu| t}\|X(t, s) \phi-X(t, s) \psi\|_{C} \quad \text { for all } t \geq s
$$

we have

$$
g(t) \leq M+N \int_{s}^{t} g(\xi) d \xi \text { for all } t \geq s
$$

where $M=e^{|\mu|(r-s)}\|\phi-\psi\|_{C}$ and $N=L e^{|\mu| r}$. Now by applying Gronwall's inequality (or precisely, a generalized version of it) we get the estimate

$$
\|X(t, s) \phi-X(t, s) \psi\|_{C} \leq K e^{\omega(t-s)}\|\phi-\psi\|_{C},
$$

where $K=e^{|\mu| r}$ and $\omega=|\mu|+L e^{|\mu| r}$. Now substituting (48) into (47) we get

$$
\begin{aligned}
\|\Delta \phi-\Delta \psi\|_{C} & \leq \sup _{-r \leq \theta \leq 0} \int_{s}^{t+\theta} e^{|\mu|(t+r-\xi)} L K e^{\omega(\xi-s)}\|\phi-\psi\|_{C} d \xi \\
& \leq e^{|\mu| r}\left(1-e^{-L e^{|\mu| r}(t-s)}\right) e^{\left(|\mu|+L e^{|\mu| r}\right)(t-s)} .
\end{aligned}
$$

Using the elementary estimates

$$
\left|1-e^{x}\right| \leq|x| e^{|x|} \text { and } t-s \leq e^{t-s}
$$


as well as $L<1$ we get

$$
\|\Delta \phi-\Delta \psi\|_{C} \leq \epsilon e^{\omega^{\prime}(t-s)}\|\phi-\psi\|_{C} \quad \text { for all } t \geq s, \phi, \psi \in C,
$$

where $\epsilon=L e^{2|\mu| r}$ and $\omega^{\prime}=1+|\mu|+e^{|\mu| r}$. The proof of the proposition is complete.

We are now in a position to apply the results achieved in Section 3 to study the instability of solutions of the evolution equation with delay (47).

Proposition 11. Let all assumptions of Proposition 10 be fulfilled. In addition let $\{T(t) \mid t \geq 0\}$ have an exponential dichotomy with nontrivial projections. Then for sufficiently small $L$ there exists an unstable integral manifold for equation (47), and consequently, the zero solution of equation (47) is unstable.

Proof. First note that the exponential dichotomy of $\{T(t) \mid t \geq 0\}$ provides an exponential dichotomy for the linear process $\{U(t, s) \mid t \geq s\}$ defined in Proposition 10. Now in view of this proposition it is sufficient to apply the results of the previous section to get the claimed assertion.

\section{ApPEndix. Uniqueness OF MiLd SOlutions}

For the reader's convenience in this appendix we present a sufficient condition for the uniqueness of mild solutions of equation (1). Since this result is primarily a minor adaptation of a result proved in [21] we only sketch the details. We first describe some assumptions on the function $f(t, x)$ in the right hand side of equation (1).

Definition 10. A function $g: \mathbb{R} \times \mathbb{R}: \rightarrow \mathbb{R}$ is said to satisfy Condition $G$ if it satisfies the following conditions:

(1) $g(t, w)$ is continuous in $w$ for each fixed $t$ and Lebesgue measurable in $t$ for each fixed $w$ and for each $r>0$ there exists a locally integrable function $L_{r}(t)$ defined on $\mathbb{R}$ such that $|g(t, w)| \leq L_{r}(t)$ for all $t \in \mathbb{R}$ and $w \in[-r, r]$

(2) $g(t, 0)=0$ and $w(t)=0$ is the maximal solution of the initial-value problem

$$
\left\{\begin{array}{l}
w^{\prime}(t)=g(t, w(t)), \quad \text { for } a<t<b \\
w(a)=0
\end{array}\right.
$$

where $a$ and $b$ are arbitrary real numbers such that $a<b$.

Proposition 12. Let the following conditions be fulfilled:

i) Equation (4) is well posed.

ii) $g(t, w)$ defined as a function on $\mathbb{R} \times \mathbb{R}$ such that

$$
[x-y,-(f(t, x)-f(t, y))] \geq g(t,|x-y|) \quad \text { for all }(t, x),(t, y) \in D(f)
$$

satisfies condition $G$.

Then for every fixed $(t, x)$ equation (1) has at most one solution. 
Proof. A minor modification of the proof of [21, Proposition 4.1].

Acknowledgement. This work was done while N. V. Minh was a fellow of the Alexander von Humboldt Foundation. The assistance of the Foundation is gratefully acknowledged. N. V. Minh would also like to express his thanks to Professors R. Nagel (Tübingen) and Yu. Latushkin (Missouri) for useful discussions and encouragement.

\section{REFERENCES}

[1] B. Aulbach, N. V. Minh and P. P. Zabreiko, Integral manifolds of a general model of evolutionary processes with impulse effects, Nonl. Anal. TMA, 23 (1994), 197-214.

[2] B. Aulbach and N. V. Minh, Semigroups and differential equations with almost periodic coefficients, to appear.

[3] V. Barbu, Nonlinear Semigroups and Differential Equations in Banach Spaces, Noordhoff, Leyden, 1976.

[4] C. K. J. Batty and V. Q. Phong, Stability of individual elements under one-parameter semigroups, Trans. Amer. Math. Soc. 322 (1990), 805-818.

[5] H. Brézis, Opérateurs maximaux monotones et semigroupes de contractions dans les espaces de Hilbert, North-Holland, Amsterdam, 1973.

[6] H. Brézis and A. Pazy, Convergence and approximation of semigroups of nonlinear operators in Banach spaces, J. Funct. Anal. 9 (1972), 63-74.

[7] S. N. Chow and H. Leiva, Existence and roughness of the exponential dichotomy for skew-product semiflows in Banach spaces, J. Differential Equations, 120 (1995), 429477.

[8] S. N. Chow, X. B. Lin and K. Lu, Smooth invariant foliations in infinite dimensional spaces, J. Differential Equations, 94 (1991), 226-291.

[9] S. N. Chow and K. Lu, Invariant manifolds for flows in Banach spaces, J. Differential Equations, 74 (1988), 285-317.

[10] W. A. Coppel, Dichotomies in Stability Theory, Lecture Notes in Math., \#629, Springer-Verlag, Berlin-New York, 1978.

[11] M. G. Crandall, Nonlinear semigroups and evolution equations governed by accretive operators, Proc. Sympos. Pure Math., \#45, Part 1, Amer. Math. Soc., 1986, 305-337.

[12] M. G. Crandall, T. M. Liggett, Generation of nonlinear transformations on general Banach spaces, Amer. J. Math. 93 (1971), 265-298.

[13] J. L. Daleckii and M. G. Krein, Stability of Solutions of Differential Equations in Banach Space, Amer. Math. Soc., Providence, 1974.

[14] W. Desch and W. Schappacher, Linearized stability for nonlinear semigroups, in Differential Equations in Banach Spaces (Favini A. and Obrecht E. eds.), Lecture Notes in Math., \#1223, Springer-Verlag, Berlin-New York, 1986, 61-73.

[15] G. Greiner, Linearized stability for hyperbolic evolution equations with semilinear boundary conditions, Semigroup Forum 38 (1989), 203-214.

[16] J. K. Hale, Asymptotic Behavior of Dissipative Systems, Amer. Math. Soc., Providence, 1988.

[17] A. Haraux, Nonlinear Evolution Equations - Global Behaviour of Solutions, Lecture Notes in Math., \#841, Springer-Verlag, Berlin-New York, 1981.

[18] D. Henry, Geometric Theory of Semilinear Parabolic Equations, Lecture Notes in Math., \#840, Springer-Verlag, Berlin-New York, 1981.

[19] E. Hille and R. Phillips, Functional Analysis and Semigroups, Amer. Math. Soc., Providence, 1957.

[20] M. Hirsch, C. Pugh and M. Shub, Invariant Manifolds, Lecture Notes in Math., \#583, Springer-Verlag, Berlin-New York, 1977.

[21] T. Iwamiya, Global existence of mild solutions to semilinear differential equations in Banach spaces, Hiroshima Math. J. 16 (1986), 499-530. 
[22] N. Kato, A principle of linearized stability for nonlinear evolution equations, Trans. Amer. Math. Soc. 347 (1995), 2851-2868.

[23] T. Kato, Perturbation Theory for Linear Operators, Springer-Verlag, Berlin-New York, 1984.

[24] H. Komatsu (ed.), Functional Analysis and Related Topics, 1991, Lecture Notes in Math., \#1540, Springer-Verlag, Berlin-New York, 1993.

[25] J. Latushkin and S. Montgomery-Smith, Evolutionary semigroups and Lyapunov theorems in Banach spaces, J. Func. Anal. 127 (1995), 173-197.

[26] J. Mallet-Paret and G. Sell, Inertial manifolds for reaction diffusion equations in higher space dimensions, J. Amer. Math. Soc. 1 (1988), 805-866.

[27] R. Martin, Nonlinear operators and differential equations in Banach spaces, WileyInterscience, New York, 1976.

[28] A. Mielke, A reduction principle for nonautonomous systems in infinite dimensional spaces, J. Differential Equations, 65 (1986), 68-88.

[29] A. Mielke, Steady flows of inviscid fluids under localized perturbations, J. Differential Equations, 65 (1986), 89-116.

[30] R. Nagel (ed.), One-parameter Semigroups of Positive Operators, Lecture Notes in Math., \#1184, Springer-Verlag, Berlin-New York, 1984.

[31] N. V. Minh, Semigroups in Qualitative Theory of Ordinary Differential Equations, in Russian, Thesis, Belorussian State Univ., Minsk, 1992.

[32] N. V. Minh, Nonlinear semigroups and nonlinear nonautonomous differential equations, in Russian, Dokl. Belorussian Acad. Sci. 37 (1993), 113-117.

[33] N. V. Minh, Semigroups and stability of nonautonomous differential equations in Banach spaces, Trans. Amer. Math. Soc., 345 (1994), 223-242.

[34] Z. Nitecki, "An Introduction to the Orbit Structure of Diffeomorphisms. MIT Press, Cambridge, 1971.

[35] S. Oharu and T. Takahashi, Locally Lipschitz continuous perturbations of linear dissipative operators and nonlinear semigroups, Proc. Amer. Math. Soc. 100 (1987), 187-194.

[36] S. Oharu and T. Takahashi, Characterization of nonlinear semigroups associated with semilinear evolution equations, Trans. Amer. Math. Soc. 311 (1989), 593-619.

[37] N. H. Pavel, Nonlinear Evolution Operators and Semigroups. Applications to Partial Differential Equations, Lecture Notes in Math., \#1260, Springer-Verlag, Berlin-New York, 1987.

[38] A. Pazy, Semigroups of Linear Operators and Applications to Partial Differential Equations, Appl. Math. Sci., \#44, Springer-Verlag, Berlin-New York, 1983.

[39] V. A. Pliss, A reduction principle in the theory of stability of motion, Izv. Acad. Nauk SSSR Mat. Ser. 28 (1964), 1297-1324.

[40] R. Rau, Hyperbolic Evolution Semigroups, Thesis, University of Tübingen, 1992.

[41] R. Rau, Hyperbolic evolutionary semigroups on vector valued function spaces, Semigroup Forum, 48 (1994), 107-118.

[42] R. Sacker and G. Sell, A spectral theory for differential systems, J. Differential Equations, 27 (1978), 320-358.

[43] R. Sacker and G. Sell, Dichotomies for linear evolutionary equations in Banach spaces, J. Differential Equations, 113 (1994), 17-67.

[44] N. Sanekata, Abstract quasi-linear equations of evolution in nonreflexive Banach spaces, Hiroshima Math. J. 19 (1989), 109-139.

[45] I. Segal, Non-linear semi-groups, Ann. Math. 78 (1963), 339-364.

[46] M. Taboada and Y. You Y, Invariant manifolds for retarded semilinear wave equations, J. Differential Equations, 114 (1994), 337-369.

[47] C. C. Travis and G. F. Webb, Existence and stability for partial functional differential equations, Trans. Amer. Math. Soc. 200 (1974), 395-418.

[48] G. F. Webb, Continuous nonlinear perturbations of linear accretive operators in Banach spaces, J. Func. Anal. 10 (1972), 191-203. 
[49] G. F. Webb, Asymptotic stability for abstract nonlinear functional differential equations, Proc. Amer. Math. Soc. 54 (1976), 225-230.

[50] K. Yosida, Functional Analysis, Springer-Verlag, berlin-New York, 1965.

Bernd Aulbach

Department of Mathematics

University of AugsBurg

D-86135 Augsburg

GERMANY

E-mail address: aulbach@math.uni-augsburg.de

NGUYen VAN Minh

Department of Mathematics

University of Augsburg

D-86135 Augsburg, GERMANY

AND

Department of Mathematics

UNIVERSITY OF TÜBINGEN

D-72076 TÜBINGEN, GERMANY

E-mail address: ming@michelangelo.mathematik.uni-tuebingen.de 


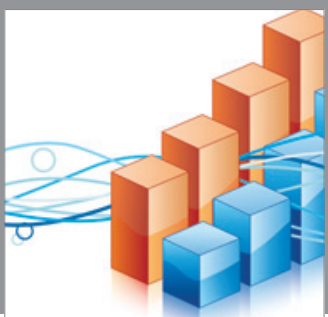

Advances in

Operations Research

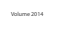

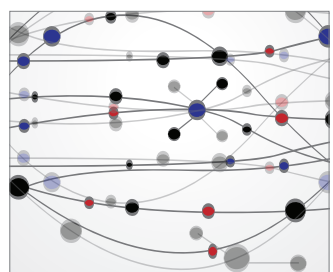

\section{The Scientific} World Journal
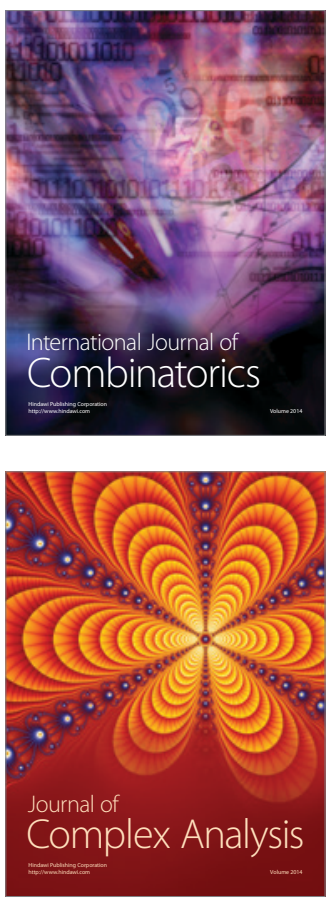

International Journal of

Mathematics and

Mathematical

Sciences
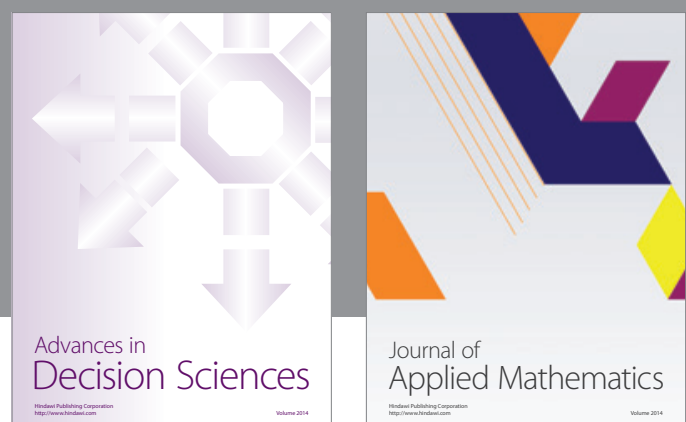

Journal of

Applied Mathematics
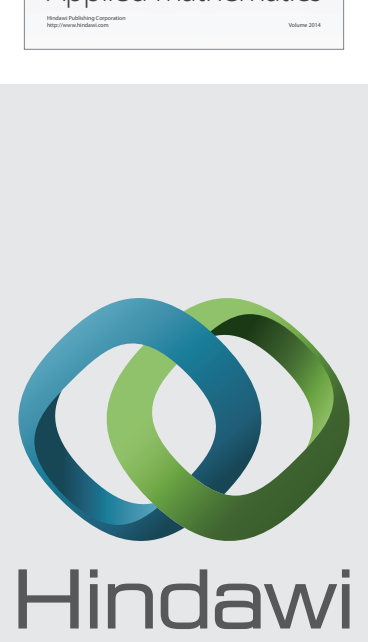

Submit your manuscripts at http://www.hindawi.com
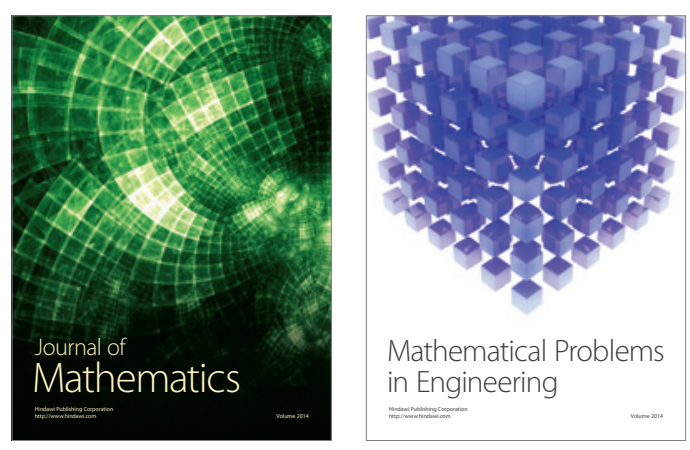

Mathematical Problems in Engineering
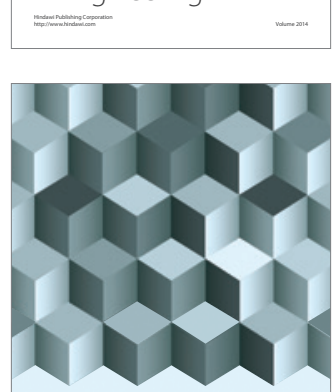

Journal of

Function Spaces
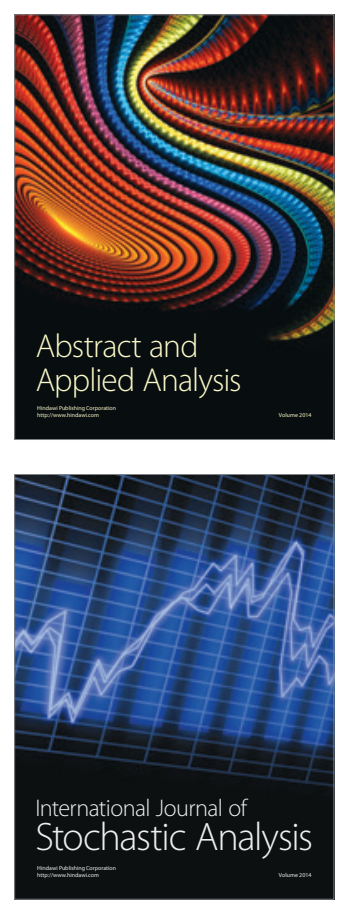

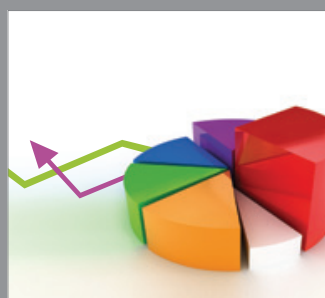

ournal of

Probability and Statistics

Promensencen
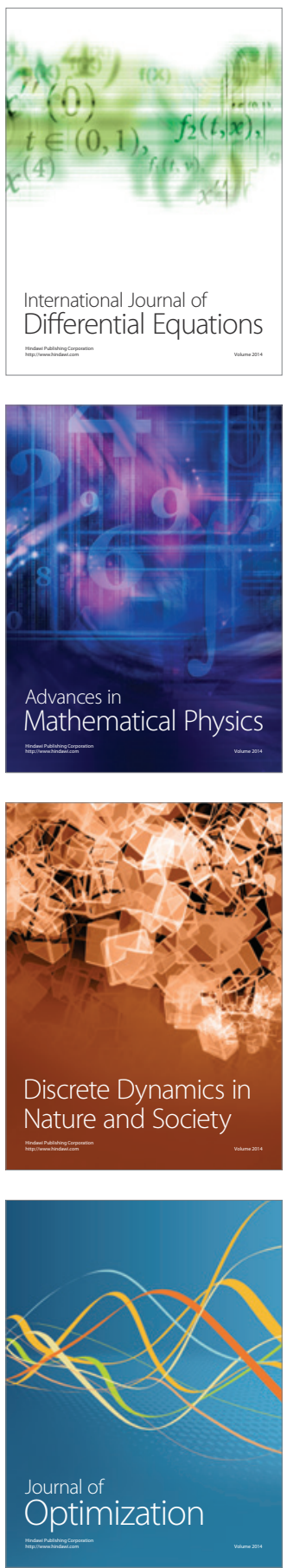